\title{
Leptophilic dark matter and the anomalous magnetic moment of the muon
}

\author{
Prateek Agrawal, ${ }^{a}$ Zackaria Chacko $^{b}$ and Christopher B. Verhaaren ${ }^{b}$ \\ ${ }^{a}$ Fermilab, \\ P.O. Box 500, Batavia, IL 60510, U.S.A. \\ ${ }^{b}$ Maryland Center for Fundamental Physics, Department of Physics, University of Maryland, \\ Physical Sciences Complex, College Park, MD 20742-4111 U.S.A. \\ E-mail: prateek@fnal.gov, zchacko@umd.edu, cver@umd.edu
}

ABSTRACT: We consider renormalizable theories such that the scattering of dark matter off leptons arises at tree level, but scattering off nuclei only arises at loop. In this framework, the various dark matter candidates can be classified by their spins and by the forms of their interactions with leptons. We determine the corrections to the anomalous magnetic moment of the muon that arise from its interactions with dark matter. We then consider the implications of these results for a set of simplified models of leptophilic dark matter. When a dark matter candidate reduces the existing tension between the standard model prediction of the anomalous magnetic moment and the experimental measurement, the region of parameter space favored to completely remove the discrepancy is highlighted. Conversely, when agreement is worsened, we place limits on the parameters of the corresponding simplified model. These bounds and favored regions are compared against the experimental constraints on the simplified model from direct detection and from collider searches. Although these constraints are severe, we find there do exist limited regions of parameter space in these simple theories that can explain the observed anomaly in the muon magnetic moment while remaining consistent with all experimental bounds.

KEYWORDS: Phenomenological Models

ARXIV EPRINT: 1402.7369 


\section{Contents}

1 Introduction 1

2 Contributions to $a_{\mu} \quad 3$

2.1 Charged mediator diagrams 3

2.2 Neutral mediator diagrams 5

3 Limits from direct detection $\quad 7$

3.1 Self-conjugate particles 8

3.2 Complex scalar DM 9

3.3 Dirac fermion DM 10

3.4 Neutral vector mediator 11

3.5 Limits 12

4 Collider constraints $\quad 13$

4.1 Neutral mediators 13

$\begin{array}{ll}\text { 4.1.1 Compositeness bounds from LEP } & 13\end{array}$

$\begin{array}{lll}\text { 4.1.2 Resonant production at LEP } & 14\end{array}$

$\begin{array}{lll}\text { 4.1.3 Light neutral mediators } & 15\end{array}$

$\begin{array}{lll}4.2 & \text { Charged mediators } & 15\end{array}$

$\begin{array}{lll}\text { 4.2.1 } & \text { Monophoton searches at LEP } & 15\end{array}$

$\begin{array}{lll}\text { 4.2.2 } & \text { Collider limits on charged mediators } & 16\end{array}$

5 Analysis of simplified models $\quad 16$

$\begin{array}{ll}5.1 \text { Real scalar dark matter } & 17\end{array}$

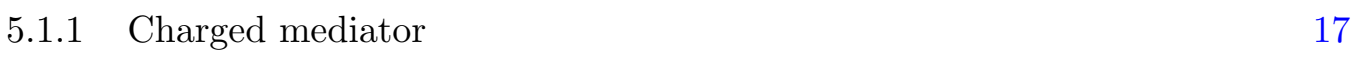

$\begin{array}{lll}\text { 5.1.2 Neutral spin-0 mediator } & 18\end{array}$

$\begin{array}{lll}5.2 & \text { Complex scalar DM } & 19\end{array}$

$\begin{array}{lll}\text { 5.2.1 Charged mediator } & 19\end{array}$

$\begin{array}{ll}5.2 .2 \text { Neutral spin-0 mediator } & 20\end{array}$

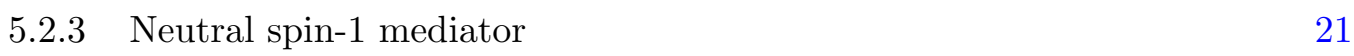

5.3 Majorana DM 21

$\begin{array}{lll}\text { 5.3.1 Charged spin-0 mediator } & 21\end{array}$

$\begin{array}{ll}\text { 5.3.2 Charged spin-1 mediator } & 22\end{array}$

5.3.3 Neutral spin-0 mediator $\quad 23$

5.3.4 Neutral spin-1 mediator 24

$\begin{array}{lll}5.4 & \text { Dirac DM } & 25\end{array}$

5.4.1 Charged spin-0 mediator 25

5.4.2 Charged spin-1 mediator 25

5.4.3 Neutral spin-0 mediator 26

$\begin{array}{ll}\text { 5.4.4 Neutral spin-1 mediator } & 27\end{array}$ 
5.5.1 Charged fermion mediator 28

5.5.2 Neutral spin-0 mediator 28

\section{Introduction}

The standard model (SM) of particle physics is in very good agreement with experiment [1]. The recent discovery of a SM-like Higgs particle at the Large Hadron Collider (LHC) [2, 3] provides further confirmation that the SM is an excellent description of the interactions of the elementary particles up to the weak scale. Nevertheless, there is now compelling experimental evidence that points to physics beyond the SM. Among the phenomena that cannot be accounted for within the SM are the non-zero values of the neutrino masses, and the existence of a baryon asymmetry.

As cosmological data has become more precise, it has become clear that more than $20 \%$ of the energy density in the universe is associated with some form of dark matter (DM) [4]. While very little is known about the precise nature of DM, a large class of well motivated theories involve particles that have interactions of weak scale strength with visible matter. These include theories where DM is composed of WIMPs, particles of weak scale mass that survive as thermal relics [5, 6], and some models of Asymmetric Dark Matter [7-17] (see [18] for a recent review and references).

Several types of experiments are involved in the search for DM. These include direct detection experiments searching for DM scattering events, indirect detection experiments searching for the products of DM annihilation such as photons and neutrinos, and colliders such as the LHC that seek to produce DM. Over the years these experiments have become increasingly sensitive, and their limits now exclude a significant part of the preferred parameter space for many WIMP DM candidates.

With the WIMP paradigm beginning to come under strain, several ideas have been put forward to explain the absence of a signal in these experiments. Perhaps the simplest possibility is that DM is leptophilic, coupling preferentially to leptons rather than to quarks [19-36]. This can help explain the null results of each of the different classes of experiments. Since the scattering of DM off nuclei now arises only at loop, the constraints from direct detection are weaker $[27,37,38]$. Because the LHC is a hadron collider, the production of particles that do not have significant couplings to quarks or gluons is suppressed, and the resulting limits are again relatively weak. The limits on leptophilic DM from indirect detection also tend to be less severe. Since the average number of photons produced in DM annihilation to electrons and muons is much smaller than in the case of hadronic final states, the constraints from continuum photons are in general weaker, for example [3948]. Limits on DM from gamma ray lines tend to be significantly weaker than the limits from continuum photons, as may be seen from model independent analyses $[49,50]$ and 
are therefore easily satisfied. Internal bremsstrahlung signatures are an interesting probe of these models [51-58], but also do not present severe constraints. Although constraints from positron flux measurements are stronger for annihilations into electrons and muons, scenarios where leptophilic DM emerges as a thermal relic remain viable [59-64].

In general, DM candidates that couple directly to SM leptons - the muon in particular - with weak scale strength are expected to have a significant impact on the anomalous magnetic moment of the muon, $a_{\mu}[58,65]$. It may therefore be possible to obtain a tighter constraint on leptophilic DM from the existing precision measurements of $a_{\mu}$ than from the experiments considered in the previous paragraph. Furthermore, with the reduction of experimental and theoretical uncertainties in the determination of $a_{\mu}$, a $3 \sigma$ discrepancy between the two has remained. The discrepancy is given by $\Delta a_{\mu} \equiv a_{\mu}^{\mathrm{Exp}}-a_{\mu}^{\mathrm{SM}}=28.7(6.3)(4.9) \times 10^{-10}[1,66-71]$, where the errors shown represent the experimental and theoretical uncertainties, respectively. The theoretical error is dominated by hadronic contributions [72-77] (further references and reviews can be found in [78, 79]). The total uncertainty including both the experimental and theoretical contributions is given by $\delta a_{\mu} \equiv 8.0 \times 10^{-10}$. If this observed anomaly is a consequence of new physics, a very interesting possibility is that it is associated with the interactions of leptons with DM. It is therefore important to identify theories of DM that can give rise to such a signal.

In this paper we consider the contributions to the anomalous magnetic moment of the muon in theories of leptophilic DM. We focus on renormalizable theories where the scattering of DM off leptons arises at tree level, but DM scattering off nuclei only arises at loop. We also assume that the interactions of $\mathrm{DM}$ respect $C P$ so that there are no corrections to the electric dipole moments of the leptons. In such a framework, the various DM candidates can be classified by their spins and by the forms of their interactions with leptons. For each case, we determine the corrections to $a_{\mu}$ that arise from these interactions. We then apply these results to several specific simplified models of DM. For concreteness, we focus on theories where a single DM candidate couples to all 3 flavors of SM leptons with equal strength.

When the DM candidate reduces the tension between the predicted value of $a_{\mu}$ in the SM and the experimental measurement, we highlight the region of parameter space favored to completely remove the discrepancy. Conversely, when agreement with experiment is worsened, we place limits on the parameters of the corresponding simplified model. These bounds and favored regions are compared against the experimental constraints on the simplified model from direct detection and collider experiments. We find that although these constraints are severe, there do exist limited regions of parameter space in these simple theories that can explain the anomaly in $a_{\mu}$ while remaining consistent with current experimental bounds. In our analysis, we do not take into account limits on the parameter space that arise from the relic abundance, since these bounds tend to be more model dependent, and are less robust than the constraints we consider. In particular, these constraints do not apply to scenarios where the dark matter is non-thermal or asymmetric.

In section 2 we begin by calculating the leading one loop contributions to $a_{\mu}$ that arise from renormalizable theories of leptophilic DM. In section 3 we determine the bounds on this scenario from direct detection and in section 4 from LEP. These results are applied to specific simplified models of DM in section 5 . 


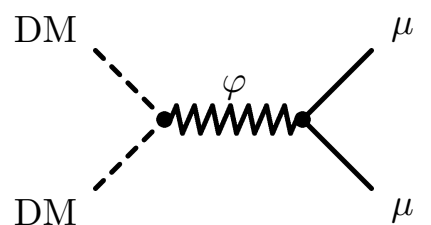

(a)

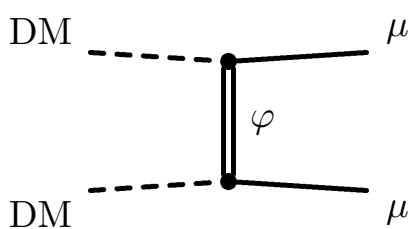

(b)

Figure 1. Two possible channels of DM to muon scattering through a mediator $\varphi$. In (a) $\varphi$ is electrically neutral and in (b) it is charged.

\section{Contributions to $a_{\mu}$}

In this section we consider the contributions to $a_{\mu}$ from different theories of leptophilic DM. Our focus is on renormalizable theories where the scattering of DM off leptons arises at tree level. These theories can be separated into two broad categories depending on whether the particle that mediates the scattering of DM off leptons is neutral or electrically charged. These two classes of theories are shown in figure 1.

These interactions can also affect the anomalous magnetic moment of the electron $a_{e}$. However, if the new physics couples universally to all the different flavors of leptons, its contributions (henceforth denoted as $\hat{a}$ ) to $a_{e}$ are much smaller than to $a_{\mu}$ and are therefore expected to easily satisfy the experimental bounds. The one important exception to this statement is the case of very light neutral mediators. Indeed, in analogy with QED, the one-loop contribution to the anomalous magnetic moment from a massless neutral mediator is equal for both electrons and muons.

In other cases, the one-loop new physics contributions to $a_{e}$ can be obtained from $a_{\mu}$ simply by replacing the mass of the muon with the mass of the electron. In the models we consider, this amounts to multiplying the result by $\frac{m_{e}^{2}}{m_{\mu}^{2}} \approx 2 \times 10^{-5}$. Note that the current uncertainty in $a_{e}$ is $\delta a_{e}=0.8 \times 10^{-12}[1,80]$, where the dominant uncertainty arises from the measurement of $\alpha$, the fine structure constant. The relative sensitivity of the two measurements can then be seen as

$$
\frac{\hat{a}_{e} / \delta_{e}}{\hat{a}_{\mu} / \delta_{\mu}} \sim \frac{\delta_{\mu}}{\delta_{e}} \times \frac{m_{e}^{2}}{m_{\mu}^{2}} \sim 0.02
$$

We see that even though it is a more precise measurement, at present $a_{e}$ is not as sensitive a probe of new physics as $a_{\mu}$, except for very light mediators.

\subsection{Charged mediator diagrams}

We begin by considering the case of DM-lepton scattering through a charged mediator, as shown in figure 1b. This scenario encompasses several different theories. In this section, it is convenient to group the different theories by the form of the interaction rather than by the type of DM. For instance, the term

$$
\mathcal{L}_{\text {vector }}=\bar{\mu} \gamma^{\nu}\left(a+b \gamma^{5}\right) F V_{\nu}+\text { h.c. }
$$




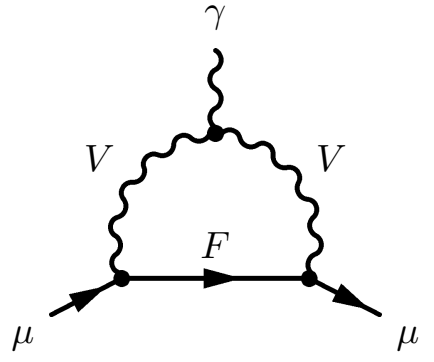

(a)

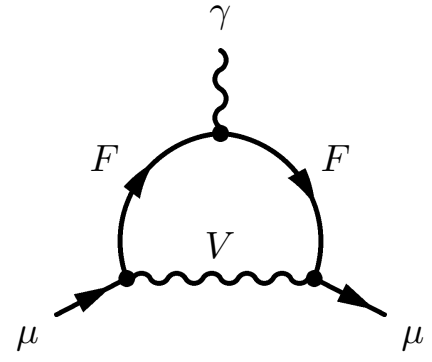

(b)

Figure 2. One loop contributions to $a_{\mu}$ involving a massive vector $V^{\mu}$ and massive fermion $F$. In (a) the DM is fermionic while in (b) it is a vector.

describes a renormalizable interaction between the muon, a massive fermion $F$ and a massive vector $V^{\nu}$. Depending on the model, either $F$ or $V^{\mu}$ constitutes the DM. The leading contribution to $a_{\mu}$ from interactions of this form arise from figure $2 \mathrm{a}$ in the case of fermionic $\mathrm{DM}$, or from figure $2 \mathrm{~b}$ for vector DM.

To keep track of each diagram's contribution to $a_{\mu}$ the labeling $\hat{a}_{\mu}^{\mathrm{Med}, \mathrm{DM}}$ will be used for each of the charged mediator contributions. The first term in the superscript refers to the spin of the mediator while the second refers to the spin of the DM. For instance the diagram in figure $2 \mathrm{a}$ is the dominant contribution to $\hat{a}_{\mu}^{V, F}$, corresponding to the case of a fermionic DM candidate whose scattering off leptons is mediated by a massive vector.

The other charged mediator interaction is of the form

$$
\mathcal{L}_{\text {scalar }}=\bar{\mu}\left(a+b \gamma^{5}\right) F S+\text { h.c. }
$$

where $S$ denotes a massive scalar. This leads to the diagram shown in figure 3 a if the DM is a fermion, or to the diagram shown in $3 \mathrm{~b}$ if the DM is a scalar.

In the diagrams considered in this subsection the mediating particle is necessarily electrically charged. Therefore, if the mediator is a fermion it must be Dirac, while if it is a scalar or a vector, it must be complex. No such restriction applies to the DM particle. Therefore, if the DM is a fermion it could be either Majorana or Dirac, while if it is a boson it could either real or complex.

The contributions to $a_{\mu}$ from these diagrams have been calculated exactly in [81] and [82]. However, as the mass of the DM $m_{\mathrm{DM}}$ and the mediator $m_{\mathrm{Med}}$ are expected to be much larger than the mass of the muon $m_{\mu}$, the leading order effects can be most clearly seen by expanding the results in powers of the small parameter $\varepsilon \equiv m_{\mu} / m_{\mathrm{Med}}$. A shorthand designating the ratio $r \equiv m_{\mathrm{DM}} / m_{\text {Med }}$ will also be used.

We first give the contributions involving the massive vector. From figure 2 a we obtain

$$
\begin{aligned}
\hat{a}_{\mu}^{V, F}=\frac{\varepsilon}{16 \pi^{2}} & \left\{\frac{\varepsilon\left(|a|^{2}+|b|^{2}\right)}{3\left(1-r^{2}\right)^{4}}\left[10-43 r^{2}+78 r^{4}-49 r^{6}+4 r^{8}+18 r^{6} \ln \left(r^{2}\right)\right]\right. \\
& \left.-\frac{r\left(|a|^{2}-|b|^{2}\right)}{\left(1-r^{2}\right)^{3}}\left[4-15 r^{2}+12 r^{4}-r^{6}-6 r^{4} \ln \left(r^{2}\right)\right]\right\}+\mathcal{O}\left(\varepsilon^{3}\right)
\end{aligned}
$$




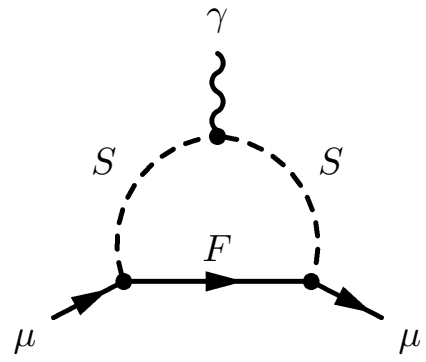

(a)

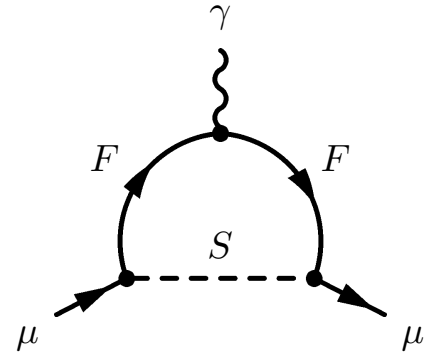

(b)

Figure 3. One loop contributions to $a_{\mu}$ involving a massive scalar $S$ and massive fermion $F$. In (a) the DM is fermionic while in (b) it is a scalar.

while from figure $2 \mathrm{~b}$ we find

$$
\begin{aligned}
\hat{a}_{\mu}^{F, V}=-\frac{\varepsilon}{16 \pi^{2} r^{2}} & \left\{\frac{\varepsilon\left(|a|^{2}+|b|^{2}\right)}{3\left(1-r^{2}\right)^{4}}\left[5-14 r^{2}+39 r^{4}-38 r^{6}+8 r^{8}+18 r^{4} \ln \left(r^{2}\right)\right]\right. \\
& \left.-\frac{|a|^{2}-|b|^{2}}{\left(1-r^{2}\right)^{3}}\left[1+3 r^{4}-4 r^{6}+6 r^{4} \ln \left(r^{2}\right)\right]\right\}+\mathcal{O}\left(\varepsilon^{3}\right) .
\end{aligned}
$$

The contributions from the scalar interactions in figures $3 \mathrm{a}$ and $3 \mathrm{~b}$ are respectively

$$
\begin{aligned}
& \hat{a}_{\mu}^{S, F}=-\frac{\varepsilon}{16 \pi^{2}}\{ \frac{\varepsilon\left(|a|^{2}+|b|^{2}\right)}{3\left(1-r^{2}\right)^{4}}\left[1-6 r^{2}+3 r^{4}+2 r^{6}-6 r^{4} \ln \left(r^{2}\right)\right] \\
&\left.+\frac{r\left(|a|^{2}-|b|^{2}\right)}{\left(1-r^{2}\right)^{3}}\left[1-r^{4}+2 r^{2} \ln \left(r^{2}\right)\right]\right\}+\mathcal{O}\left(\varepsilon^{3}\right), \\
& \hat{a}_{\mu}^{F, S}=\frac{\varepsilon}{16 \pi^{2}}\left\{\frac{\varepsilon\left(|a|^{2}+|b|^{2}\right)}{3\left(1-r^{2}\right)^{4}}\left[1-6 r^{2}+3 r^{4}+2 r^{6}-6 r^{4} \ln \left(r^{2}\right)\right]\right. \\
&\left.+\frac{|a|^{2}-|b|^{2}}{\left(1-r^{2}\right)^{3}}\left[1-4 r^{2}+3 r^{4}-2 r^{4} \ln \left(r^{2}\right)\right]\right\}+\mathcal{O}\left(\varepsilon^{3}\right) .
\end{aligned}
$$

In all of the above relations the $r$ dependent functions contained within the square brackets are never negative and hence never change the sign of the contribution. Therefore, in each case the sign of the correction to $a_{\mu}$ is completely determined by the relative sizes of $a$ and $b$. An interesting feature pointed out by [83] in the context of supersymmetric theories, but which is completely general, is that the contribution to $a_{\mu}$ from a coupling to the muon that respects chiral symmetry, $a= \pm b$, is suppressed by order $\varepsilon$ relative to the results of an interaction that violates this symmetry.

\subsection{Neutral mediator diagrams}

We now consider DM-lepton scattering mediated by a neutral particle, as shown in figure 1a. The interactions between the muon and a vector or a scalar mediator are of the same form as in (2.2) and (2.3) respectively, but with the fermion $F$ replaced by a muon $\mu$. Because the contributions to $a_{\mu}$ in these models are insensitive to the nature of the DM particle, we simply label the contributions by the spin of the mediator, i.e. $\hat{a}_{\mu}^{V}$ for a vector mediator. 


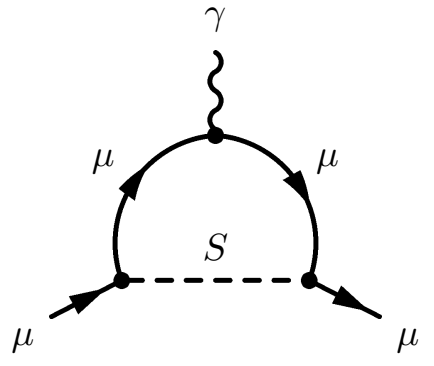

(a)

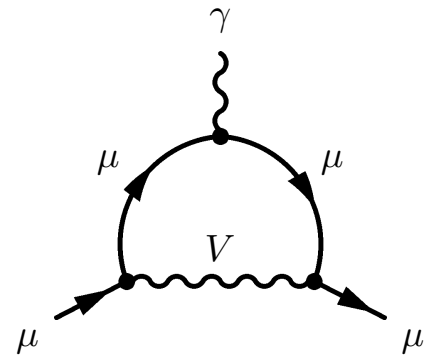

(b)

Figure 4. One loop contributions from models with neutral mediators to $a_{\mu}$ by (a) a scalar $S$ and (b) a vector $V^{\mu}$.

The interactions of the neutral mediator models are nearly identical to the charged mediator models, and in principle the exact expressions from the calculation above can be adapted for this case. However, the expansion in terms of the ratio of the muon mass to the charged particle running in the loop, in this case the muon itself, is obviously not valid here. We continue using $\varepsilon$ to denote the ratio of masses of the muon and the neutral mediator, and present the leading order result in this parameter,

$$
\begin{aligned}
& \hat{a}_{\mu}^{V}=\frac{\varepsilon^{2}}{4 \pi^{2}}\left[-\frac{2}{3}\left(|a|^{2}+|b|^{2}\right)+\left(|a|^{2}-|b|^{2}\right)\right]+\mathcal{O}\left(\varepsilon^{3}\right), \\
& \hat{a}_{\mu}^{S}=\frac{\varepsilon^{2}}{8 \pi^{2}}\left[\frac{1}{3}\left(|a|^{2}+|b|^{2}\right)-\left(\frac{3}{2}+\ln \varepsilon^{2}\right)\left(|a|^{2}-|b|^{2}\right)\right]+\mathcal{O}\left(\varepsilon^{3}\right) .
\end{aligned}
$$

Notice that the contribution from interactions that respect chiral symmetry is no longer suppressed by an additional power of $\varepsilon$ relative to the contribution from couplings that violate this symmetry. In the case of a scalar mediator, the chiral symmetry violating effects are only logarithmically enhanced, while in the case of a vector mediator they are of the same order. When the mediator is lighter than or comparable in mass to the muon, the small $\varepsilon$ approximation does not apply, and we use the full expressions for our numerical analysis of constraints.

The muon $g-2$ is sensitive only to couplings of the DM and mediators to the muon. However, other constraints depend on the flavor structure of the couplings to all leptons. Therefore, to compare the muon $g-2$ with these limits, we must make some assumptions about the flavor structure of the models under consideration. The non-observation of flavor-changing processes in charged leptons puts strong constraints on the couplings of DM to leptons. We focus on theories where a single DM candidate couples to all 3 flavors of SM leptons with equal strength. Accordingly, we assume that there is a separate charged mediator corresponding to each lepton flavor, and that there is no mixing between different flavors of mediators. Another possibility that also satisfies flavor bounds, but which we will not consider in this paper, is to have a separate DM species corresponding to each flavor of lepton, but only a single mediator [37]. 


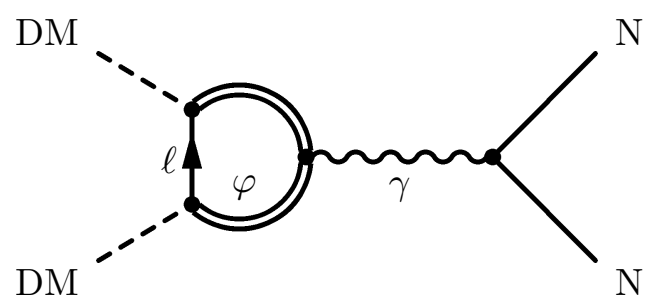

(a)

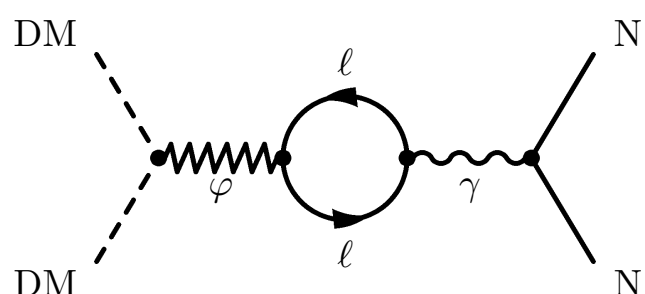

(b)

Figure 5. Leading order processes for DM nucleon scattering for (a) charged and (b) neutral mediators. Note that there is a second diagram at the same order with the lepton $\ell$ coupling to the photon in the charged mediator case.

\section{Limits from direct detection}

In this section we determine the limits on leptophilic DM from direct detection experiments. In these theories scattering off nuclei only arises at loop level. Therefore, we expect that the direct detection bounds will most significantly constrain theories where DM-nucleon scattering is spin-independent, and arises at one loop. In our analysis we use the recently released LUX results [84] to generate the bounds.

In figure 5 we see the leading contributions to a DM particle $\chi$ scattering with a nucleus $N$, arising from a photon exchange. The contributions mediated by the exchange of the Higgs or the $Z$ are suppressed by small lepton Yukawa couplings [85]. In general, the direct detection cross section receives contributions from all the SM leptons running in the loop. As stated above, we focus on theories where the couplings of the DM to each flavor of the SM leptons are equal.

The velocity of incident DM particles is expected to be small $\left(v / c \sim 10^{-3}\right)$, which implies that the momentum transfer in direct detection experiments will be of order 10$50 \mathrm{MeV}$. Since the momentum transfer in this process is so small, it is convenient to work in an effective theory with the charged particles in the loop that mediate scattering integrated out. The effective theory will be consistent provided that the momentum transfer in the scattering process is smaller than the mass of the particles in the loop. Although this condition is not strictly satisfied in the case of the electron, it is straightforward to correct for this. In the case of charged mediators, in the effective theory the DM candidate couples directly to the photon through an effective vertex.

Similar considerations apply to the neutral mediator case, provided the mass of the neutral mediator is more than the typical momentum transfer. Of course, since DMnucleon scattering arises via mixing of the mediator with the photon, only the neutral vector contributes. The mixing is radiatively generated by a loop diagram that involves particles charged under both the photon and the new vector boson. At low energies, the only particles in the loop that contribute to mixing are the charged leptons of the SM. The loop diagram is logarithmically divergent, and needs to be regulated. For concreteness, we assume that the diagram is cut off at a scale $\Lambda_{V}$, which we take to be of order the weak scale. After integrating out the mediator and the charged leptons, the DM again couples to the photon through an effective vertex at low energies. 
The case of very light neutral mediator $(m \lesssim 30 \mathrm{MeV})$ is slightly more subtle. We can no longer integrate out the mediator for typical scattering at direct detection experiments. In this case, it is more convenient to eliminate the mixing between the mediator and the photon by a redefinition of the photon field, which leads to a direct coupling of the SM quarks to the neutral mediator. This translates into a tree-level interaction of the DM with quarks in the low energy theory, mediated by the new vector boson.

It follows that the direct detection bounds can be translated into limits on the effective operators that couple DM to the photon, and then into constraints on the parameters of the theory. These constraints are expected to be most significant for theories that give rise to effective operators that generate spin-independent DM-nucleon scattering at one loop. In what follows we consider the DM candidates of different spins and identify the relevant effective operators that lead to spin-independent DM-nucleon interactions. Those theories with no such operator are not expected to be significantly constrained by the current limits from direct detection.

The effective operators generated by integrating out the particles in the loop respect the symmetries of the underlying theory. Therefore, we need only consider effective operators that respect these symmetries. In particular, since we are restricting our discussion to interactions that are invariant under $C P$, we need only consider effective operators that respect $C P$.

\subsection{Self-conjugate particles}

It can be shown quite generally that in the case of self-conjugate (real scalar, Majorana fermion, real vector) leptophilic DM, the direct detection signals are suppressed. A selfconjugate particle couples to a single photon only through an anapole moment [86-91]. In particular, for $C P$-conserving interactions, the scattering amplitude of the DM particles with the quark electromagnetic current $\left(\bar{q} \gamma^{\mu} q\right)$ can be written in the following form,

$$
\mathcal{M} \sim\left(k^{2} s_{\mu}-(k \cdot s) k_{\mu}\right) \frac{1}{k^{2}} \bar{q} \gamma^{\mu} q .
$$

Here $p_{1}$ and $p_{2}$ are the incoming and outgoing DM momenta respectively, and $k=p_{2}-p_{1}$ is the momentum transfer. The quantity $s_{\mu}$ depends on the spins and momenta of the incoming and outgoing DM particles. As an example, for a Majorana fermion, $s^{\mu}=$ $\left\langle\chi\left(p_{2}\right)\left|\bar{\chi} \gamma^{\mu} \gamma^{5} \chi\right| \chi\left(p_{1}\right)\right\rangle$. The second term above does not contribute to the scattering process (due to Ward identities). The $k^{2}$ in the first term cancels against the photon propagator, giving rise to a contact interaction with the quark current. For direct detection, this implies that the scattering amplitude with the nucleus is given by

$$
\mathcal{M} \sim \sum_{q}\left\langle N_{f}\left|Q \bar{q} \gamma^{\mu} q\right| N_{i}\right\rangle s_{\mu} .
$$

Since the anapole moment is $P$-odd (a symmetry respected by the electromagnetic coupling), it follows that this contact interaction will lead to $p$-wave scattering amplitudes that are suppressed in the non-relativistic limit. Therefore, we expect that the direct detection constraints on self-conjugate DM particles coupling to the nucleons via a photon will be relatively weak. 
These conclusions may also be directly obtained from a study of the possible effective operators. We limit ourselves to operators of dimension up to six. It is easily seen that there are no operators that couple real scalar DM to a single photon. Such an operator would schematically contain two DM field factors $\chi^{2}$, the photon field strength $F_{\mu \nu}$ (or its dual), and derivatives. The antisymmetry of $F^{\mu \nu}$, however, makes any operator of this type vanish identically.

As is well-known, the bilinears $\bar{\chi} \gamma^{\mu} \chi$ and $\bar{\chi} \sigma_{\mu \nu} \chi$ both vanish identically for Majorana fermions. The only surviving bilinear is proportional to $\bar{\chi} \gamma^{\mu} \gamma^{5} \chi$ which gives velocity suppressed matrix elements with the electromagnetic current of the nucleus.

Finally for real vector DM the dimension four operator $\chi_{\mu} \chi_{\nu} F^{\mu \nu}$ vanishes by the antisymmetry of the Maxwell tensor. We can apply two derivatives as in the real scalar case, but find that the resulting operator violates $C P$. This leads us to consider the operator

$$
\mathcal{O}_{V}=\partial_{\mu} \chi^{\alpha} \partial_{\alpha} \chi_{\nu} F_{\sigma \rho} \varepsilon^{\mu \nu \sigma \rho}
$$

Note that any other choice of contraction of indices vanishes either due to the antisymmetry of $\varepsilon^{\mu \nu \sigma \rho}$ or the on-shell constraint $\partial_{\alpha} \chi^{\alpha}=0$. The resulting amplitude is

$$
\mathcal{M} \sim \sum_{q}\left\langle N_{f}\left|Q \bar{q} \gamma_{\rho} q\right| N_{i}\right\rangle \frac{1}{k^{2}} \varepsilon^{\mu \nu \sigma \rho} k_{\sigma}\left(p_{2}+p_{1}\right)_{\mu} k_{\alpha}\left[\epsilon_{\nu}^{*}\left(p_{2}\right) \epsilon^{\alpha}\left(p_{1}\right)-\epsilon_{\nu}\left(p_{1}\right) \epsilon^{* \alpha}\left(p_{2}\right)\right]
$$

where the $\epsilon_{\nu}(p)$ are polarization vectors of $\chi_{\nu}$. The $k_{\sigma} k_{\alpha}$ suppression is overcome by the photon propagator. However, the epsilon-tensor contraction of $\left(p_{1}+p_{2}\right)$ with $\bar{q} \gamma^{\mu} q$ vanishes in the limit that the velocities of the incoming particles tend to zero. Therefore, this amplitude is suppressed in the non-relativistic limit.

We conclude that leptophilic DM particles which are real scalars, Majorana fermions or real vectors are not generally expected to be significantly constrained by direct detection searches. The DM candidates that give rise to sizable spin-independent cross sections in the non-relativistic limit are complex scalars and Dirac fermions. We study those cases in more detail.

\subsection{Complex scalar DM}

If the DM is a complex scalar there is a non-vanishing effective operator, namely

$$
\mathcal{O}_{S}=A_{S} i\left[\partial_{\mu} \chi^{*} \partial_{\nu} \chi-\partial_{\nu} \chi^{*} \partial_{\mu} \chi\right] F^{\mu \nu}
$$

Note that if we replace $F^{\mu \nu}$ by $F_{\alpha \beta} \varepsilon^{\alpha \beta \mu \nu}$ the corresponding operator is odd under $C P$. From $\mathcal{O}_{S}$ we find the complex scalar DM contribution to the direct detection amplitude,

$$
\mathcal{M}_{\mathcal{O}_{S}}=-A_{S} e \sum_{q}\left\langle N_{f}\left|Q \bar{q} \gamma^{\nu} q\right| N_{i}\right\rangle\left(p_{2}+p_{1}\right)_{\nu} \equiv \tilde{\lambda} \sum_{q}\left\langle N_{f}\left|Q \bar{q} \gamma^{\nu} q\right| N_{i}\right\rangle\left(p_{2}+p_{1}\right)_{\nu}
$$

where the sum over $q$ denotes a sum over quark bilinears, $p_{1}$ and $p_{2}$ the DM momenta before and after scattering, and $Q$ is the charge each quark in units of $e$. 


\subsection{Dirac fermion DM}

For a Dirac fermion DM, the lowest dimension operator we can write down which preserves $C P$ and gauge invariance is

$$
\mathcal{O}=i A_{5} \bar{\chi} \sigma_{\mu \nu} \chi F^{\mu \nu} .
$$

However, this effective operator violates the chiral symmetry associated with $\chi$, and will not be generated in theories where the couplings of DM respect this symmetry. When present this operator leads to the amplitude

$$
\mathcal{M}=2 A_{5} e \sum_{q}\left\langle N_{f}\left|Q \bar{q} \gamma^{\nu} q\right| N_{i}\right\rangle \frac{k^{\mu}}{k^{2}} \bar{u}\left(p_{2}\right) \sigma_{\mu \nu} u\left(p_{1}\right)
$$

where $k=p_{2}-p_{1}$ is the momentum transfer. This amplitude gives rise to dipole-charge and dipole-dipole interactions between the DM and the nucleus [92, 93]. The dipole-charge interaction is enhanced by the atomic number $Z$ for heavy nuclei, and hence dominates. It is important to note that the recoil spectrum arising from this interaction is distinct from that of the familiar contact interaction, and simply scaling the WIMP-nucleon cross section limit does not give accurate results in this case.

In the simplified models we consider, the dominant contribution arises at dimension six. At this order, we have two possible $C P$-even operators,

$$
\begin{aligned}
& \mathcal{O}_{1}=\left[\bar{\chi} \gamma^{\mu}\left(c+d \gamma^{5}\right) \partial^{\nu} \chi+\text { h.c. }\right] F_{\mu \nu}, \\
& \mathcal{O}_{2}=\left[i \bar{\chi} \gamma^{\mu}\left(c+d \gamma^{5}\right) \partial^{\nu} \chi+\text { h.c. }\right] F^{\sigma \rho} \varepsilon_{\mu \nu \sigma \rho}
\end{aligned}
$$

where the factors of $i$ have been chosen to make the effective theory coefficients $c$ and $d$ real. These operators were considered in appendix A of [37] for the case of charged scalar mediator.

The above operators lead to the amplitudes

$$
\begin{aligned}
& \mathcal{M}_{\mathcal{O}_{1}}=-e c \sum_{q}\left\langle N_{f}\left|Q \bar{q} \gamma^{\mu} q\right| N_{i}\right\rangle \bar{u}\left(p_{2}\right) \gamma_{\mu} u\left(p_{1}\right), \\
& \mathcal{M}_{\mathcal{O}_{2}}=4 i e d \sum_{q}\left\langle N_{f}\left|Q \bar{q} \gamma_{\alpha} q\right| N_{i}\right\rangle \frac{1}{k^{2}} \bar{u}\left(p_{2}\right)\left[m_{\chi} \sigma^{\alpha \beta} k_{\beta}+\frac{i}{2} k^{2} \gamma^{\alpha}\right] u\left(p_{1}\right) .
\end{aligned}
$$

where we have neglected velocity suppressed terms. Notice that $\mathcal{O}_{2}$ leads to interactions of the charge-charge, dipole-charge and dipole-dipole form. We use the charge-charge interaction to set limits. The generic amplitude for this can be parametrized as

$$
\mathcal{M}=\tilde{\lambda} \sum_{q}\left\langle N_{f}\left|Q \bar{q} \gamma_{\alpha} q\right| N_{i}\right\rangle \bar{u}\left(p_{2}\right) \gamma^{\alpha} u\left(p_{1}\right) .
$$

Since the argument of logarithm is large, a leading-log approximation (as employed in [27]) suffices to place limits on these models. 


\subsection{Neutral vector mediator}

We take a brief detour to consider the case of the neutral vector mediator separately, since it has a few distinct qualitative features. We choose the case of Dirac fermion DM for illustration. To begin, we assume that both the mediator and the leptons are heavier than the typical momentum transfer at direct detection experiments. This is obviously not correct for the electron and very light mediators, and we will subsequently correct for this.

We first consider the case where the mediator is heavier than all the leptons. Just above the scale of the mediator mass, the relevant terms in the effective Lagrangian are given by,

$$
\mathcal{L}_{\text {eff }}=\lambda \bar{\chi} \gamma^{\mu} \chi V_{\mu}+\lambda \bar{\ell} \gamma^{\mu} \ell V_{\mu}-\frac{1}{2} m_{V}^{2} V^{\mu} V_{\mu}+e \bar{\ell} \gamma^{\mu} \ell A_{\mu}-\frac{1}{4} F_{\mu \nu} F^{\mu \nu}-\frac{1}{4} F_{\mu \nu}^{\prime} F^{\prime \mu \nu}+\tilde{\epsilon} F_{\mu \nu}^{\prime} F^{\mu \nu} .
$$

Since the leptons are charged under both the $A_{\mu}$ and $V_{\mu}$, they contribute to the kinetic mixing between the photon and the neutral mediator at one loop. To set conservative limits, we assume that this constitutes the dominant contribution to $\tilde{\epsilon}$, so that $\tilde{\epsilon}$ in the leading log approximation is given by,

$$
\tilde{\epsilon}=\frac{\lambda e}{24 \pi^{2}} \sum_{l} \log \frac{m_{V}^{2}}{\Lambda_{V}^{2}} .
$$

For concreteness, we take the cut-off $\Lambda_{V}$ to be $1 \mathrm{TeV}$.

The mediator can be integrated out using its equation of motion.

$$
\left(\partial^{2} g^{\mu \nu}-\partial^{\mu} \partial^{\nu}+m_{V}^{2} g^{\mu \nu}\right) V_{\mu}=2 \tilde{\epsilon} \partial_{\mu} F^{\mu \nu}+\lambda \bar{\chi} \gamma^{\mu} \chi+\lambda \bar{\ell} \gamma^{\mu} \ell
$$

Thus, below the mediator mass, the relevant terms at leading order are

$$
\mathcal{L}_{\text {eff }}=\frac{\lambda^{2}}{m_{V}^{2}} \bar{\chi} \gamma^{\mu} \chi \bar{\ell} \gamma^{\mu} \ell+e \bar{\ell} \gamma^{\mu} \ell A_{\mu}+\frac{2 \tilde{\epsilon} \lambda}{m_{V}^{2}} \bar{\chi} \gamma^{\nu} \chi \partial_{\mu} F^{\mu \nu}-\frac{1}{4} F_{\mu \nu} F^{\mu \nu}
$$

With this Lagrangian, the DM couples to the photon through the higher dimensional operator directly, but also via a loop of leptons. The second contribution is logarithmically divergent, which is to say that the coupling of DM to photons continues to run. Below the mass of the leptons, we are only left with,

$$
\mathcal{L}_{\text {eff }}=\frac{2 \epsilon \lambda}{m_{V}^{2}} \bar{\chi} \gamma^{\nu} \chi \partial_{\mu} F^{\mu \nu}-\frac{1}{4} F_{\mu \nu} F^{\mu \nu}
$$

The coefficient $\epsilon$ includes logarithms from running between $\Lambda$ to $m_{V}$, as well as $m_{V}$ to $m_{l}$. In fact, from the full theory calculation these terms are easily seen to combine into a single logarithm of the ratio of scales $m_{\ell} / \Lambda_{V}$.

$$
\epsilon=\frac{\lambda e}{24 \pi^{2}} \sum_{l} \log \frac{m_{\ell}^{2}}{\Lambda_{V}^{2}}
$$

If the mediator is lighter than the leptons, then the effective Lagrangian below the lepton masses contains a kinetic-mixing of the mediator with the photon.

$$
\mathcal{L}_{\text {eff }}=\lambda \bar{\chi} \gamma^{\mu} \chi V_{\mu}-\frac{1}{2} m_{V}^{2} V^{\mu} V_{\mu}-\frac{1}{4} F_{\mu \nu} F^{\mu \nu}-\frac{1}{4} F_{\mu \nu}^{\prime} F^{\prime \mu \nu}+\epsilon F_{\mu \nu}^{\prime} F^{\mu \nu}
$$


where $\epsilon$ is defined in eq. (3.19). Below this scale, the DM coupling to photons does not run. One the mediator is integrated out, we again generate the same operator as above.

$$
\mathcal{L}_{\text {eff }}=\frac{2 \epsilon \lambda}{m_{V}^{2}} \bar{\chi} \gamma^{\nu} \chi \partial_{\mu} F^{\mu \nu}-\frac{1}{4} F_{\mu \nu} F^{\mu \nu}
$$

Thus, we see that the effective operators generated in the neutral mediator case have the same form as in the charged mediator case, and hence the results from that analysis can be applied here as well.

We finally address the assumption that the leptons and the mediator masses are heavier than the momentum transfer in direct detection experiments. At LUX, the momentum transfer corresponding to the energy thresholds is around $|q| \sim 30 \mathrm{MeV}$. Therefore, the logarithms appearing in the expression for $\epsilon$, eq. (3.19), are cut off by $|q|$ in the case of the electron, rather than by the electron mass.

If the neutral mediator mass is below the typical scattering energies, $|q| \sim 30 \mathrm{MeV}$, it cannot be integrated out. However, we can eliminate the kinetic mixing in eq. (3.20) via a field redefinition $A_{\mu} \rightarrow A_{\mu}+2 \epsilon V_{\mu}$. After this redefinition, quarks pick up couplings to the vector mediator, via which DM can scatter off nuclei at direct detection experiments. Since the mediator is very light, the propagator $1 /\left(q^{2}-m^{2}\right)$ is dominated by $1 / q^{2}$, and hence the scattering cross section scales as $1 / q^{4}$. For setting limits in this regime, we assume that the scattering is dominated by $|q| \sim 30 \mathrm{MeV}$. This level of approximation will suffice for our purposes.

\subsection{Limits}

The differential cross section for charge-charge interactions takes the form

$$
\frac{d \sigma}{d \vec{k}^{2}}=\frac{Z^{2} \widetilde{\lambda}^{2}}{4 \pi v^{2}} F^{2}\left(\vec{k}^{2}\right)
$$

where $Z$ is the charge number of the nucleus and $F\left(\vec{k}^{2}\right)$ is the charge form factor of the nucleus. The quantity $\tilde{\lambda}$ is the coefficient of the charge-charge interaction operators in eqs. (3.6) and (3.13). This expression applies to both cases above.

In order to make connection with experimental limits, we evaluate the zero momentum transfer cross section, which is obtained by integrating (3.22) evaluated at $\vec{k}=0$.

$$
\sigma_{N}^{0}=\frac{\mu_{N}^{2} Z^{2}}{\pi} \widetilde{\lambda}^{2}
$$

where $\mu_{N}$ is the reduced mass of the DM-nucleus system. Further, since limits are reported in terms of the DM-nucleon cross section, we rescale the DM-nucleus cross section,

$$
\sigma_{n}^{0}=\sigma_{N}^{0} \frac{\mu_{n}^{2}}{\mu_{N}^{2}} \frac{1}{A^{2}}=\frac{\mu_{n}^{2} Z^{2}}{\pi A^{2}} \widetilde{\lambda}^{2}
$$

where $\mu_{n}$ is the reduced mass of the DM nucleon system and $A$ is the number of nucleons in the nucleus. Thus, given an effective Lagrangian, limits can be placed on $\widetilde{\lambda}$ using (3.24). In section 5 , we calculate the coefficient $\widetilde{\lambda}$ in the context of a set of simplified models, and use the equation above to place limits on the parameter space. 


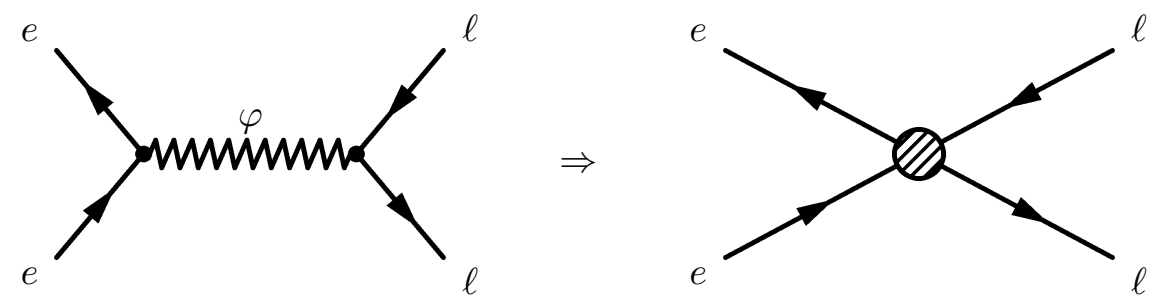

Figure 6. Effective four-lepton interaction generated upon integrating out a heavy neutral mediator.

\section{Collider constraints}

In this section we study collider constraints on leptophilic dark matter models. In our analysis, we assume that the DM candidate couples with equal strength to all three flavors of leptons. In this scenario, DM particles can be pair-produced at LEP, in association with hard initial state radiation and significant missing transverse energy. This is called the monophoton signal. Existing analyses focus on the case of fermionic DM [94].

Neutral mediators can be resonantly produced at LEP and other $e^{+} e^{-}$colliders if they are light enough. If they are heavier than $208 \mathrm{GeV}$, the LEP energy threshold, the LEP limits on four-lepton operators apply, the so-called "compositeness bounds" [1]. Charged mediators can be pair-produced at colliders. Studies of direct slepton pair-production can be translated into limits on these models. In general, these constraints on parameter space are comparable to, and often stronger than, the restrictions from $a_{\mu}$.

\subsection{Neutral mediators}

\subsubsection{Compositeness bounds from LEP}

Models with neutral mediators heavier than $208 \mathrm{GeV}$ are constrained by severe limits on four-lepton contact operators, the compositeness bounds [1,95]. These operators are generated at tree-level when the neutral mediator that couples the DM to leptons is integrated out (see figure 6). The bounds are expressed as limits on the new physics scale $\Lambda$ that appears as the coefficient suppressing these higher-dimensional operators. The operators can be parametrized as [96]

$$
\mathcal{L}=\frac{4 \pi}{(1+\delta) \Lambda^{2}}\left[\eta_{L L} \bar{e}_{L} \gamma^{\mu} e_{L} \bar{\ell}_{L} \gamma_{\mu} \ell_{L}+\eta_{R R} \bar{e}_{R} \gamma^{\mu} e_{R} \bar{\ell}_{R} \gamma_{\mu} \ell_{R}+\eta_{L R} \bar{e}_{L} \gamma^{\mu} e_{L} \bar{\ell}_{R} \gamma_{\mu} \ell_{R}+\eta_{R L} \bar{e}_{R} \gamma^{\mu} e_{R} \bar{\ell}_{L} \gamma_{\mu} \ell_{L}\right]
$$

where coefficients $\eta$ characterize the relative contributions of different chiralities. The factor $\delta$ is 1 when $\ell=e$ and 0 otherwise.

We now write the neutral mediator models in the form of (4.1) for ease of comparison. When the mediator is a vector $V^{\mu}$ we have the interaction

$$
\mathcal{L}=\frac{\lambda}{2} \bar{\ell} \gamma_{\mu}\left(\cos \phi+\sin \phi \gamma^{5}\right) \ell V^{\mu}
$$

where $\lambda$ is a real parameter and $\phi$ a mixing angle that parametrizes the relative strengths 


\begin{tabular}{|c|cccc|c|}
\hline \multirow{2}{*}{ Bound } & \multicolumn{4}{|c|}{ Operators } & Limit \\
\cline { 2 - 5 } & $\eta_{L L}$ & $\eta_{R R}$ & $\eta_{L R}$ & $\eta_{R L}$ & $\Lambda(\mathrm{TeV})$ \\
\hline $\mathrm{VV}$ & -1 & -1 & -1 & -1 & 20.0 \\
$\mathrm{AA}$ & -1 & -1 & 1 & 1 & 18.1 \\
$\mathrm{LR}+\mathrm{RL}$ & 0 & 0 & -1 & -1 & 14.5 \\
\hline
\end{tabular}

Table 1. Summary of compositeness bounds on various four-lepton operators from LEP [97] arising from a neutral spin-1 and spin-0 mediators. Shown are the bounds for various combination of operators. In all cases the reported $\Lambda^{-}$bounds apply.

of vector and axial-vector interactions. This leads to the effective operator

$$
\begin{gathered}
\mathcal{O}_{V}=-\frac{\lambda^{2}}{4 m_{V}^{2}}\left[(1-\sin 2 \phi) \bar{e}_{L} \gamma^{\mu} e_{L} \bar{\ell}_{L} \gamma_{\mu} \ell_{L}+(1+\sin 2 \phi) \bar{e}_{R} \gamma^{\mu} e_{R} \bar{\ell}_{R} \gamma_{\mu} \ell_{R}\right. \\
\left.+\cos 2 \phi \bar{e}_{L} \gamma^{\mu} e_{L} \bar{\ell}_{R} \gamma_{\mu} \ell_{R}+\cos 2 \phi \bar{e}_{R} \gamma^{\mu} e_{R} \bar{\ell}_{L} \gamma_{\mu} \ell_{L}\right] .
\end{gathered}
$$

We compare the above operator with the bounds given in [97] for the pure vector $\phi=0$ and pure axial vector $\phi=\frac{\pi}{2}$ cases. The combined bounds from all leptons (assuming flavor universality), $e^{+} e^{-} \rightarrow \ell^{+} \ell^{-}$, leads to the most stringent constraints. The sign of the fourlepton operator coefficient is relevant due to its interference with the SM background. The coefficient of all the operators that we consider is negative, hence the $\Lambda^{-}$bounds reported in [97] are relevant, and are shown in table 1.

For a spin-0 mediator $\varphi$, we treat the scalar and pseudoscalar interactions separately. The interaction Lagrangians are

$$
\mathcal{L}_{S}=\lambda \bar{\ell} \ell \varphi \quad \mathcal{L}_{P S}=i \lambda \bar{\ell} \gamma^{5} \ell \varphi,
$$

where $\lambda$ is, in general, complex. The only resulting effective operator which fits the form of (4.1) is identical in both cases,

$$
\mathcal{O}_{S / P S}=-\frac{2|\lambda|^{2}}{8 m_{\varphi}^{2}}\left[\bar{e}_{R} \gamma^{\mu} e_{R} \bar{e}_{L} \gamma_{\mu} e_{L}\right]
$$

Thus, for both the scalar and pseudoscalar interactions we can use the combined Bhabha scattering bound from the recent results [97] (see also [98]).

In obtaining these limits we have assumed that the mass of the neutral mediator is larger than the center-of-mass energy of the collisions. Therefore, if the mediator is lighter than the LEP CM energy, $208 \mathrm{GeV}$, the bounds obtained from compositeness are not directly applicable.

\subsubsection{Resonant production at LEP}

When the mass of the mediator is below the LEP threshold, $208 \mathrm{GeV}$, it can be resonantly produced. It can subsequently decay into pair of leptons with a characteristic Breit-Wigner resonance shape in the lepton pair invariant mass distribution. These bounds can be ameliorated by forcing the dominant decays of the mediator to invisible or otherwise hidden final states. This typically requires introducing significant complexity to the new physics model. 
There are no available model independent studies for a leptophilic $Z^{\prime}$ model at LEP. Limits exist in the context of specific models, which assume a specific pattern of couplings [97]. A $Z^{\prime}$ of mass less than $209 \mathrm{GeV}$ is ruled out unless the coupling is less than about $10^{-2}$. A general analysis is likely to lead to a much stronger limit. However, this conservative limit itself will be seen to be the strongest constraint for mediator masses as low as a few $\mathrm{GeV}$.

\subsubsection{Light neutral mediators}

If the neutral mediator is very light, then even a small coupling with the leptons can give observable effects in $(g-2)_{\mu}$. Such light mediators have a variety of constraints, which we briefly review here. For a more detailed treatment of this topic, we refer to a recent review [99].

High intensity electron beams are dumped on to fixed targets. The SM particles are stopped in a shield, but very weakly interacting neutral mediators can escape this shield and their decays to pair of leptons can be observed. The beam dump experiments at Fermilab and SLAC [100-102] have been reanalyzed in the context of light neutral mediators [103]. The constraints apply for a range of couplings - small enough to escape the shielding but large enough to yield an appreciable decay rate.

High luminosity $e^{+} e^{-}$colliders can produce the neutral mediator radiatively $e^{+} e^{-} \rightarrow$ $\phi \gamma$, where $\phi$ is the neutral mediator [103-105]. The decay $\phi \rightarrow \mu^{+} \mu^{-}$provides the strongest constraint for mediator masses between several hundred $\mathrm{MeV}$ and a few $\mathrm{GeV}$. We use the reinterpreted constraints derived from BaBar data performed in [106], which was carried out for a vector mediator. For the same value of coupling, the spin- 0 mediator production cross section within the acceptance region is lower by roughly a factor of two. We scale the limits on the coupling of the spin-0 neutral mediator appropriately to reflect this fact.

The electron $g-2$ measurement is also sensitive to light neutral mediators and provides the strongest bounds on this scenario for mediator masses between a few $\mathrm{MeV}$ and several hundred MeV. A number of proposed experiments aim to probe the region of weakly coupling light mediators in the future.

\subsection{Charged mediators}

\subsubsection{Monophoton searches at LEP}

For DM masses less than half of the LEP center of mass (CM) energy, pairs of DM particles can be produced in association with a photon, $e^{+} e^{-} \rightarrow \chi \chi \gamma$.

The existing monophoton analyses focus only on fermionic DM, with the mediator heavier than the LEP threshold. Thus, these analyses put constraints on four-fermion contact operators involving the electron and DM fields using experimental limits on the process $e^{+} e^{-} \rightarrow \chi \chi \gamma$. The present limits in the literature only apply to a restricted set of operators. For the simplified models that we will consider, the following Fierz rearrangements will be relevant,

$$
\bar{\mu}\left(1+\gamma^{5}\right) \chi \bar{\chi}\left(1-\gamma^{5}\right) \mu=\frac{1}{2}\left[\bar{\mu} \gamma^{\mu} \gamma^{5} \mu \bar{\chi} \gamma_{\mu} \gamma^{5} \chi-\bar{\mu} \gamma^{\mu} \mu \bar{\chi} \gamma_{\mu} \chi-\bar{\mu} \gamma^{\mu} \mu \bar{\chi} \gamma_{\mu} \gamma^{5} \chi+\bar{\mu} \gamma^{\mu} \gamma^{5} \mu \bar{\chi} \gamma_{\mu} \chi\right]
$$




$$
\bar{\mu} \gamma^{\mu}\left(1+\gamma^{5}\right) \chi \bar{\chi} \gamma_{\mu}\left(1+\gamma^{5}\right) \mu=\bar{\mu} \gamma^{\mu} \gamma^{5} \mu \bar{\chi} \gamma_{\mu} \gamma^{5} \chi+\bar{\mu} \gamma^{\mu} \mu \bar{\chi} \gamma_{\mu} \chi+\bar{\mu} \gamma^{\mu} \mu \bar{\chi} \gamma_{\mu} \gamma^{5} \chi+\bar{\mu} \gamma^{\mu} \gamma^{5} \mu \bar{\chi} \gamma_{\mu} \chi
$$

Of the four contact operators that appear after these rearrangements only the first two (vector-vector and axial vector-axial vector) are considered in the LEP analysis. We therefore use the more stringent of these two (the vector-vector term) for Dirac DM. In essence, we are assuming that interference between the terms will not affect the bound significantly. For Majorana DM the vector bilinear vanishes identically and so we use the axial vector-axial vector bound.

\subsubsection{Collider limits on charged mediators}

Current collider limits exist only for scalar mediators. LEP rules out charged mediators lighter than about a $100 \mathrm{GeV}$. The charged scalar mediators can also be pair-produced at the LHC, each of which then decay to a lepton and DM. The signature is then a pair of opposite-sign, like-flavor leptons with missing transverse energy. After Run 1, LHC puts constraints on the production of charged scalars. The relevant bounds are presented in the context of supersymmetry, for pair production of sleptons - charged scalars carrying flavor. These analyses directly apply to charged scalar mediator case [107]. The relevant bounds for the models we consider are the "right-handed slepton" bounds, where the scalar does not carry SU(2) quantum numbers. In the case where the scalar mediator carries SU(2) charge is more strongly constrained due to its enhanced production cross section. The bounds also depend on the DM mass, and get weaker in the region where the dark matter mass is close to the mediator mass due to reduced missing transverse energy.

\section{Analysis of simplified models}

In this section we consider specific simplified models of leptophilic DM. We classify the different theories based on the spin of the DM, the spin and charge of the mediator, and the form of the interaction. A similar approach for couplings of DM to quarks was adopted in $[108,109]$, and more recently in [110-112].

For each simplified model the parameter space can be separated into regions favored or disfavored by their effects on $\Delta a_{\mu}$. When applicable, limits from direct detection and collider experiments are also considered. It is convenient to present the results in terms of the parameter $\Lambda$, defined as $m_{\mathrm{Med}} / \lambda$, where $m_{\mathrm{Med}}$ is the mediator mass and $\lambda$ the mediator couplings to leptons.

When the contribution to $a_{\mu}$ is negative the tension between theory and experiment is increased. In this scenario, we require the new physics contribution to $a_{\mu}$ be no larger than twice the uncertainty in $\Delta a_{\mu}$. In other words, we require that the "wrong sign" contributions be indistinguishable from the combined experimental and theoretical uncertainties at two standard deviations, $\left|\hat{a}_{\mu}\right| \leq 2 \delta a_{\mu}$. As $\delta a_{\mu}$ is reduced these bounds become stronger.

When the sign of $\hat{a}_{\mu}$ is positive the DM contribution improves the agreement between theory and experiment. In these cases we identify the region of parameter space that eliminates the discrepancy, so that $a_{\mu}$ agrees with the experimentally measured value to within $2 \delta a_{\mu}$. Specifically, we shade regions between the $\hat{a}_{\mu}$ values of $44.7 \times 10^{-10}$ and $12.7 \times 10^{-10}$. In these cases, as $\delta a_{\mu}$ is reduced these bands become more narrow about their central values. 
The collider limits on charged mediators depend sensitively on their transformation properties under the electroweak $\mathrm{SU}(2)_{\mathrm{L}} \times \mathrm{U}(1)_{\mathrm{Y}}$ gauge group. In general, the mediator and DM candidate could arise from any of several $\mathrm{SU}(2)$ representations, or even arise as an admixture of different representations. In this section, when considering simplified models with charged mediators, we restrict our analysis to those theories where the mediator and DM carry no charge under $\mathrm{SU}(2)_{\mathrm{L}}$. Under this assumption chiral symmetry violating couplings of the DM to muons are forbidden. The only source of violation of the chiral symmetry is then the muon mass. Further, it allows us to limit our analysis to simplified models where the DM couples only to the right-handed muons. In the subsequent formulae we will set $a=\frac{\lambda}{2}, b= \pm \frac{\lambda}{2}$, with the choice of sign corresponding to the right-handed chiral projectors for muons. Then, it can be seen from equations (2.4)-(2.7) that each of the charged mediator $\hat{a}_{\mu}$ contributions is a function of three dimensionless parameters, namely $\varepsilon, r$ and $\lambda$, and appears with a definite sign.

In the case of neutral mediators, the contribution to $a_{\mu}$ depends only on the coupling of the mediator to the muon, and is independent of the form of the interactions of the mediator with DM. The limits on neutral mediators from the LEP compositeness bounds are also independent of the coupling to DM. In general, we find that the compositeness bounds are much more restrictive than the monophoton bounds. Therefore, except in those cases for which the direct detection bounds are relevant, the limits on scenarios with a neutral mediator are independent of the details of the DM candidate. We now turn to analysing each simplified model in detail.

\subsection{Real scalar dark matter}

\subsubsection{Charged mediator}

We begin with the case of a real scalar DM candidate $\chi$ and a charged mediator $F$, which is a fermion. The relevant interaction is

$$
\mathcal{L}=\frac{\lambda}{2} \bar{\mu}\left(1-\gamma^{5}\right) F \chi+\text { h.c. }
$$

Employing (2.7) to obtain the contribution to $a_{\mu}$ we find

$$
\frac{1}{\Lambda^{2}} \equiv\left(\frac{|\lambda|}{m_{F}}\right)^{2}=\frac{96 \pi^{2}(1-r)^{4} \hat{a}_{\mu}^{F, S}}{m_{\mu}^{2}\left(1-6 r^{2}+3 r^{4}+2 r^{6}-6 r^{4} \ln r^{2}\right)}
$$

with $r=m_{\chi} / m_{F}$.

In figure 7 we plot the region of parameter space for which this model removes the $g-2$ anomaly to within $2 \sigma$. This scenario is not significantly constrained by the current limits from direct detection. The monophoton analyses in the literature have only been performed for fermionic DM, and are not directly applicable to this model. From the plot we see that for small values of $r$, the preferred values of $\Lambda$ lie between 50 and $95 \mathrm{GeV}$. For $r$ values closer to 1 the preferred range runs from about 35 to $65 \mathrm{GeV}$.

The gauge quantum numbers of the fermion $F$, and the associated collider signals, are identical to those of a slepton in supersymmetric theories. The limits on the slepton mass from direct slepton pair production at LEP stand at about $100 \mathrm{GeV}$. There exist stronger 


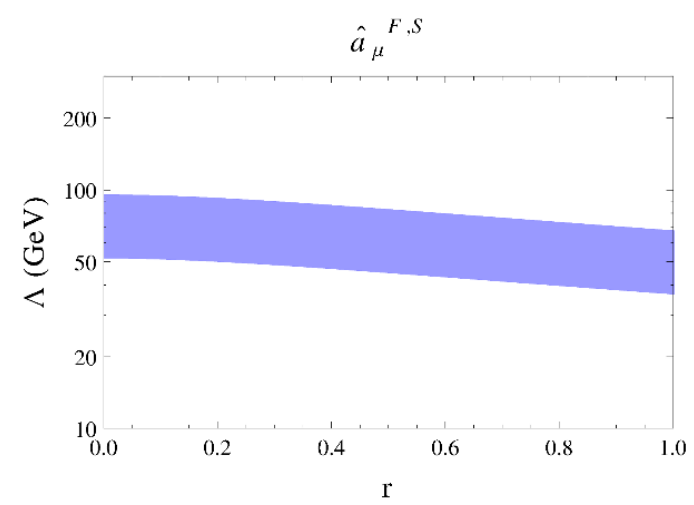

Figure 7. We see the favored band (in blue) for real scalar DM mediated by a charged fermion $F$ as a function of $r=m_{\mathrm{DM}} / m_{F}$.

bounds from the LHC for light DM masses [107]. Given that the pair production cross section is generally larger for fermions than for bosons, it is quite likely that the limits on the mass of $F$ are at least at the same level. This would imply that the region of parameter space where the $g-2$ anomaly is explained is disfavored by LEP, unless the coupling $\lambda$ is large, $\lambda \gtrsim 1$. However, a more careful analysis is required in order to validate this conclusion. We leave this for future work.

\subsubsection{Neutral spin-0 mediator}

We now turn to the case of a real spin-0 neutral mediator, $S$. A few comments are in order. Clearly, a coupling of the form $\bar{\ell} \ell S$ is not consistent with gauge invariance under $\mathrm{SM} \mathrm{SU}(2)$ if $S$ is a singlet. In principle, this problem can be avoided if $S$ arises as a linear combination of the SM Higgs and a singlet, but the theory would no longer be leptophilic since $S$ would also couple to quarks.

A potential UV completion is to add to the theory a heavy scalar $\mathrm{SU}(2)$ doublet $\widehat{H}$ which has no VEV, and couples only to leptons. After electroweak symmetry breaking the neutral components of $H$ can mix with a real singlet scalar $\widehat{S}$ via a coupling with the SM Higgs $H$. A light state $S$ with couplings to leptons can then emerge as a linear combination of the singlet $\widehat{S}$ and the neutral components of the doublet $\widehat{H}$. The relevant interactions have the schematic form

$$
\left(\hat{\lambda} \bar{L} \widehat{H} E+\kappa \widehat{S} H^{\dagger} \widehat{H}+\text { h.c. }\right)+M^{2} \widehat{H}^{\dagger} \widehat{H}
$$

where $L$ is the $\mathrm{SU}(2)$ doublet $\mathrm{SM}$ lepton and $E$ the SM singlet. The $C P$ transformation properties of $S$ depend on whether the coupling $\kappa$ is real or complex. We assume for concreteness that the coupling matrix $\hat{\lambda}$ is universal and flavor diagonal in the lepton mass basis.

The form of the low energy Lagrangian depends on the $C P$ properties of the scalar $S$. For real scalar DM, there is no $C P$-conserving coupling of the DM with a single pseudoscalar at the renormalizable level. Thus, we only consider the scalar mediator in this case. The Lagrangian is of the form

$$
\mathcal{L}_{S}=\lambda \bar{\mu} \mu S
$$




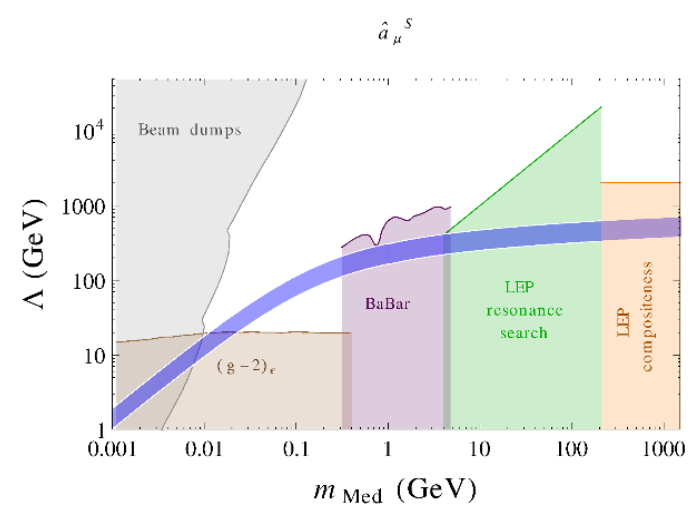

Figure 8. Plots of favored regions of parameter space for real scalar DM with interactions mediated by a neutral scalar $S$ as a function of $m_{M e d}$. The shaded blue region corresponds to the range of parameters favored to set $\Delta a_{\mu}=0$ to within $2 \sigma$. We also show bounds on light neutral scalars as colored shaded regions (compositeness bounds, resonant production at LEP, BaBar and beam dumps, and the electron $g-2)$.

Using (2.9) we find the leading contributions to $a_{\mu}$ for the scalar mediator. The contribution is written as

$$
\left(\frac{|\lambda|}{m_{S}^{2}}\right)^{2}=\frac{32 \pi^{2} \hat{a}^{S}}{m_{\mu}^{2} \ln \left(\frac{m_{S}^{2}}{m_{\mu}^{2}}\right)}
$$

In figure 8 we plot, over a range of mediator masses, the region of parameter space favored by the scalar interaction. We also plot the corresponding compositeness bound from LEP for heavy mediators. We also show bounds from resonant production at LEP and at BaBar for lower mediator masses. We see from the figure that these constraints rule out the region of parameter space where the scalar interaction can account for the observed discrepancy in $a_{\mu}$, except a small region of parameter space where the mediator is light, between about $10 \mathrm{MeV}$ and $300 \mathrm{MeV}$. This scenario is not significantly constrained by current direct detection experiments.

The case of a neutral vector mediator need not be considered. The reason is that in a renormalizable theory, real scalar DM cannot couple to a spin-1 neutral mediator without violating $C P$. There is therefore no simplified model of this type that meets our criteria.

\subsection{Complex scalar DM}

\subsubsection{Charged mediator}

We proceed to complex scalar DM $\chi$. As before, we begin with a charged fermion, denoted by $F$, as the mediator. The interaction Lagrangian takes the form

$$
\mathcal{L}=\frac{\lambda}{2} \bar{\mu}\left(1-\gamma^{5}\right) F \chi+\text { h.c. }
$$

As in the case of the real scalar (2.7) leads to

$$
\left(\frac{|\lambda|}{m_{F}}\right)^{2}=\frac{96 \pi^{2}(1-r)^{4} \hat{a}_{\mu}^{F, S}}{m_{\mu}^{2}\left(1-6 r^{2}+3 r^{4}+2 r^{6}-6 r^{4} \ln r^{2}\right)}
$$




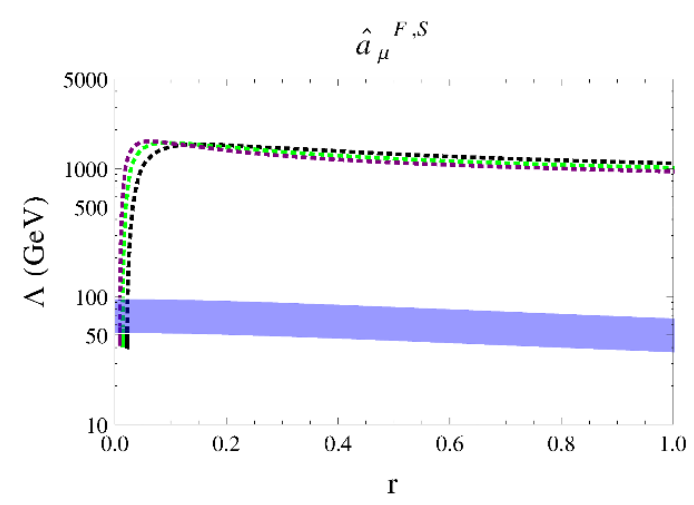

Figure 9. Regions of favored parameter space compared with bounds from direct detection and compositeness for a complex scalar DM mediated by a charged fermion. The constraints are shown in terms of $r=m_{\mathrm{DM}} / m_{\mathrm{Med}}$. The direct detection bounds are shown as dotted lines, denoting a 250 (black), 400 (green), or $600 \mathrm{GeV}$ (brown) mediator mass.

where $r=m_{\chi} / m_{F}$.

The monophoton analyses in the literature do not apply to this model. However, as shown in section 3 , this theory is constrained by direct detection. The parameter $\widetilde{\lambda}$ can be obtained by calculating the contributing diagram in the effective theory with the heavy mediator integrated out, in the leading logarithm approximation. Expressing the result in the form of (3.22) this defines $\tilde{\lambda}$ which is found to be

$$
\widetilde{\lambda}=\frac{\lambda^{2} \alpha}{12 \pi^{2} m_{\phi}^{2}}\left[\ln \frac{m_{\tau}^{2}}{m_{\phi}^{2}}+\ln \frac{m_{\mu}^{2}}{m_{\phi}^{2}}+\ln \frac{|k|^{2}}{m_{\phi}^{2}}\right]
$$

where $\alpha$ is the fine structure constant. In this calculation we have worked to zeroth order in the small parameter $k^{2} / m_{\ell}^{2}$, except in the case of the electron loop. For this diagram, we instead work to zeroth order in $m_{e}^{2} / k^{2}$, and this leads to the dependence on $|k|$ of the final result. When bounds are set we use the value $|k|=30 \mathrm{MeV}$.

In figure 9 we have overlaid these bounds on the region of parameter space which for which this model removes the $g-2$ anomaly to within $2 \sigma$. The three bands shown in the figure correspond to mediator masses of 250 (black), 400 (green), and $600 \mathrm{GeV}$ (brown). We see that the direct detection limits exclude almost the entire region, unless the DM mass lies below about $5 \mathrm{GeV}$. We further expect that a recast analysis will show that the limits on direct slepton searches at LEP disfavor the entire band, except for fairly large values of the coupling $\lambda$.

\subsubsection{Neutral spin-0 mediator}

Next, we consider a spin- 0 neutral mediator $S$. The interaction Lagrangian is of the form

$$
\mathcal{L}_{S}=\lambda \bar{\mu} \mu S
$$

for scalar mediators.

Since the direct detection constraints on this scenario are not significant, the analysis for neutral spin-0 mediators performed for the real scalar DM applies to this case as well. The results are shown in figure 8 . 


\subsubsection{Neutral spin-1 mediator}

In the case of a neutral spin-1 mediator $V^{\nu}$, the interaction Lagrangian takes the form

$$
\mathcal{L}=\frac{i \lambda}{2}\left(\chi^{*} \partial_{\nu} \chi-\chi \partial_{\nu} \chi^{*}\right) V^{\nu}+\frac{\lambda}{2} \bar{\mu} \gamma^{\mu}\left(\cos \phi+\sin \phi \gamma^{5}\right) \mu V_{\mu}
$$

Then (2.8) leads to

$$
\left(\frac{\lambda}{m_{V}}\right)^{2}=\frac{16 \pi^{2} \hat{a}_{\mu}^{V}}{m_{\mu}^{2}\left(\cos 2 \phi-\frac{2}{3}\right)} .
$$

Over the domain of $\phi$, this expression takes on both positive and negative values. We choose two specific cases, $\phi=0$, vector coupling and $\phi=\pi / 2$, the axial vector coupling, as benchmarks. There are also additional constraints from direct detection. Axial vector mediators give no contribution, so these bounds only apply to the vector $(\phi=0)$ case. The DM-nucleon scattering amplitude can be expressed in the form of eq. (3.22). In the leading logarithm approximation we obtain

$$
\widetilde{\lambda}=\frac{\lambda^{2} \alpha}{6 \pi m_{V}^{2}}\left[\ln \frac{m_{\tau}^{2}}{\Lambda_{V}^{2}}+\ln \frac{m_{\mu}^{2}}{\Lambda_{V}^{2}}+\ln \frac{|k|^{2}}{\Lambda_{V}^{2}}\right] .
$$

Here $\Lambda_{V}$ is the scale at which the logarithmic divergence is cut off. For concreteness, we take $\Lambda_{V}=1 \mathrm{TeV}$.

In figure 10 we show the favored band of parameter space for the vector case, and compare it to bounds from direct detection, and from resonant collider production. We see that the entire region of parameter space that explains the anomaly in $a_{\mu}$ is excluded by direct detection experiments if the DM particle is accessible to them $\left(m_{D M} \gtrsim 5 \mathrm{GeV}\right)$. Otherwise, a small window of mediator masses between about 10 and $300 \mathrm{MeV}$ is still allowed. For the axial-vector, the direct detection bounds do not apply, therefore the muon $g-2$ measurement is the most sensitive in the same mediator mass range.

\subsection{Majorana DM}

\subsubsection{Charged spin-0 mediator}

Turning to fermionic DM we begin by considering a Majorana fermion $\chi$ whose interactions with leptons are mediated by charged scalars $\phi_{l}$. The interaction Lagrangian takes the form

$$
\mathcal{L}=\frac{\lambda}{2} \bar{\mu}\left(1-\gamma^{5}\right) \chi \phi_{l}+\text { h.c. }
$$

The contribution to $a_{\mu}$ can be obtained from (2.6) and leads to the relation

$$
\left(\frac{|\lambda|}{m_{\phi}^{2}}\right)^{2}=-\frac{96 \pi^{2} \hat{a}_{\mu}^{S, F}(1-r)^{4}}{m_{\mu}^{2}\left(1-6 r^{2}+3 r^{4}+2 r^{6}-6 r^{4} \ln r^{2}\right)}
$$

where $r=m_{\chi} / m_{\phi}$. The sign of the contribution increases the tension between theory and experiment. This implies that $a_{\mu}$ can be used to place limits on this model. The bounds on this model from direct detection are not expected to be significant. There are constraints, 

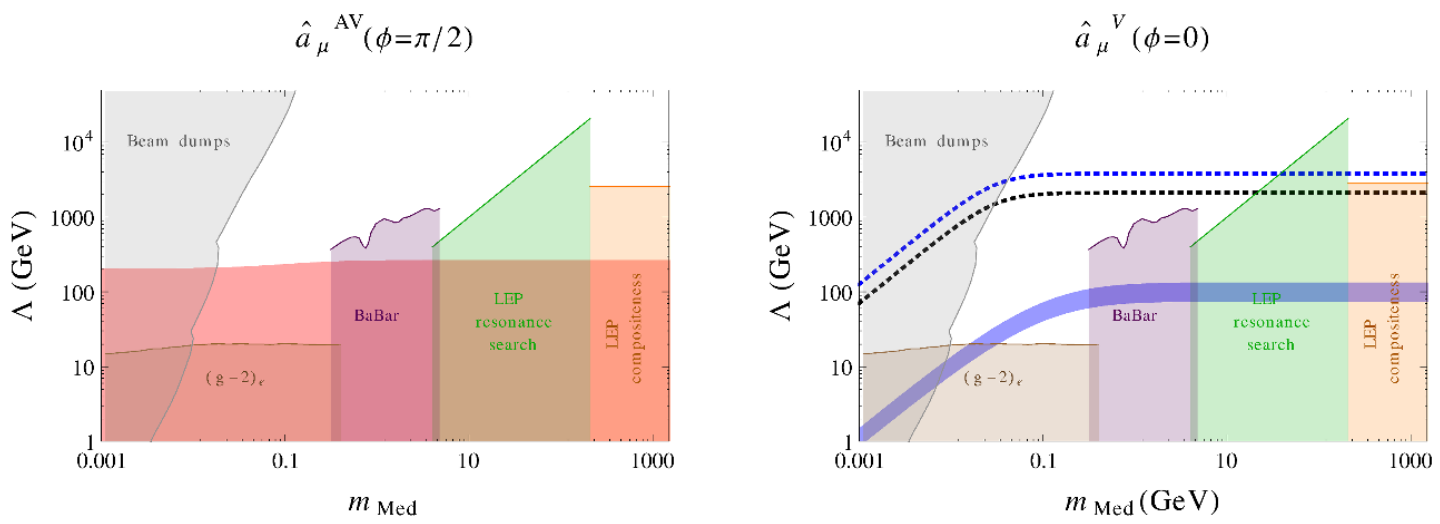

Figure 10. Limits on complex scalar DM mediated by a neutral spin-1 particle with vector interactions as a function of $m_{M e d}$ for a axial-vector mediator (left) and for a vector mediator (right). For the vector mediator, we also show direct detection bounds for two different DM masses $\left(m_{D M}=10 \mathrm{GeV}\right.$ (blue dotted) and $90 \mathrm{GeV}$ (black dotted)).

however, from the LEP monophoton searches. We compare these limits to those arising from $a_{\mu}$ in figure 11a. The three dashed lines correspond to $250 \mathrm{GeV}$ (black), $400 \mathrm{GeV}$ (green), and $600 \mathrm{GeV}$ (brown) mediators.

From the figure we see that as the mass of the mediator increases the monophoton limits become weaker, and eventually become less sensitive than the bounds from $a_{\mu}$. The $g-2$ bound restricts $\Lambda$ to be greater than $75 \mathrm{GeV}$ when $r=0.5$ and greater than $85 \mathrm{GeV}$ when $r=0.1$. Note, however, that the spin and gauge quantum numbers of $S$, and its decay modes, are identical to those of a slepton in supersymmetric theories. Therefore, the right-handed slepton bounds from LEP and the LHC apply, and are shown in figure 11b. For $\lambda=1$, the lower bound on the slepton mass from LEP and the LHC provides a stronger constraint on this model than $a_{\mu}$.

\subsubsection{Charged spin-1 mediator}

Next, we consider mediation by charged vectors $V_{l}^{\nu}$. The interaction Lagrangian is given by

$$
\mathcal{L}=\frac{\lambda}{2} \bar{\mu} \gamma_{\nu}\left(1+\gamma^{5}\right) \chi V_{l}^{\nu}+\text { h.c. }
$$

The contribution to $a_{\mu}$ can be obtained from (2.4), and leads to the relation

$$
\left(\frac{|\lambda|}{m_{V}^{2}}\right)^{2}=\frac{96 \pi^{2} \hat{a}_{\mu}^{V, F}(1-r)^{4}}{m_{\mu}^{2}\left(10-43 r^{2}+78 r^{4}-49 r^{6}+4 r^{8}+18 r^{6} \ln r^{2}\right)}
$$

where $r=m_{\chi} / m_{V}$. This expression can be used to determine the region of parameter space favored to remove the $g-2$ anomaly. While the direct detection bounds on this model are not expected to be significant, there are constraints from LEP monophoton searches that we compare to this favored region in figure 12. In the figure the LEP monophoton bounds are plotted for $250 \mathrm{GeV}$ (black), $400 \mathrm{GeV}$ (green), and $600 \mathrm{GeV}$ (brown) mediators. We see from the plot that the values of $\Lambda$ between 165 and $285 \mathrm{GeV}$ fall within the favored band. Within this range LEP bounds disfavor DM masses lighter than about $15 \mathrm{GeV}$, but there remains a significant region of parameter space that can explain the discrepancy in $a_{\mu}$. 


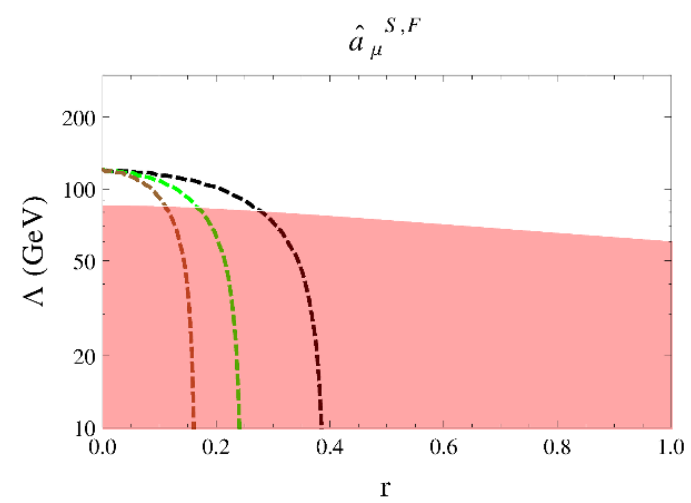

(a)

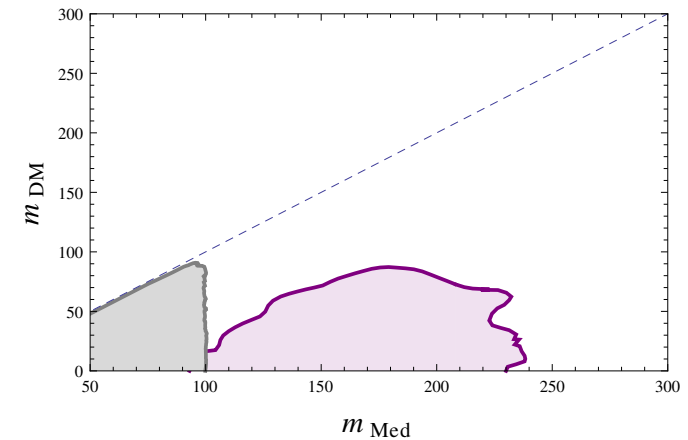

(b)

Figure 11. (a) The $g-2$ bound on Majorana DM with a charged scalar mediator as a function of $r=m_{\mathrm{DM}} / m_{\mathrm{Med}}$. Dashed lines indicate the monophoton bounds from LEP, and are plotted for 250 (black), 400 (green), and $600 \mathrm{GeV}$ (brown) mediators. (b) LHC (purple) and LEP (gray) constraints on charged scalars as a function of the mediator and DM masses.

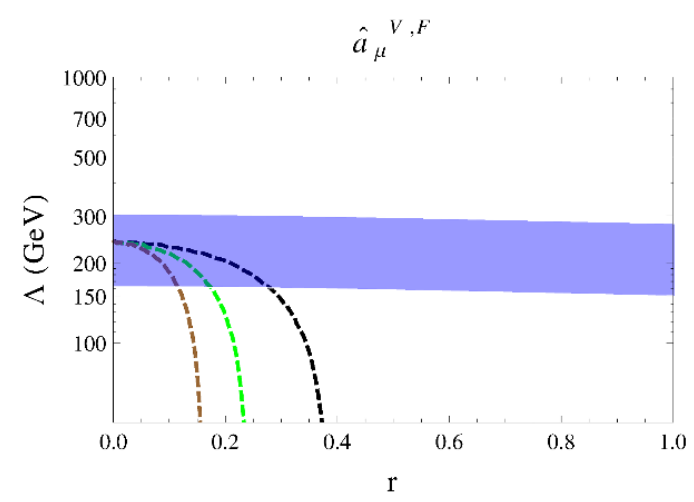

Figure 12. Constraints on Majorana DM with a charged spin-1 mediator as a function of $r=$ $m_{\mathrm{DM}} / m_{\mathrm{Med}}$. We show region favored to set $\Delta a_{\mu}=0$ to within $2 \sigma$. Dashed lines indicate the monophoton bounds from LEP, and are plotted for 250 (black), 400 (green), and $600 \mathrm{GeV}$ (brown) mediators.

\subsubsection{Neutral spin-0 mediator}

When mediation occurs through a neutral spin-0 particle $S$, the Lagrangian takes the form

$$
\mathcal{L}_{S}=\lambda \bar{\mu} \mu S
$$

We only consider the scalar mediator since there is no $C P$-conserving coupling of a pseudoscalar to a Majorana fermion at the renormalizable level. While the current limits from direct detection on leptophilic Majorana fermion DM are weak, the other limits on a neutral scalar mediator continue to apply. The results in figure 8 again show the most stringent bounds. 


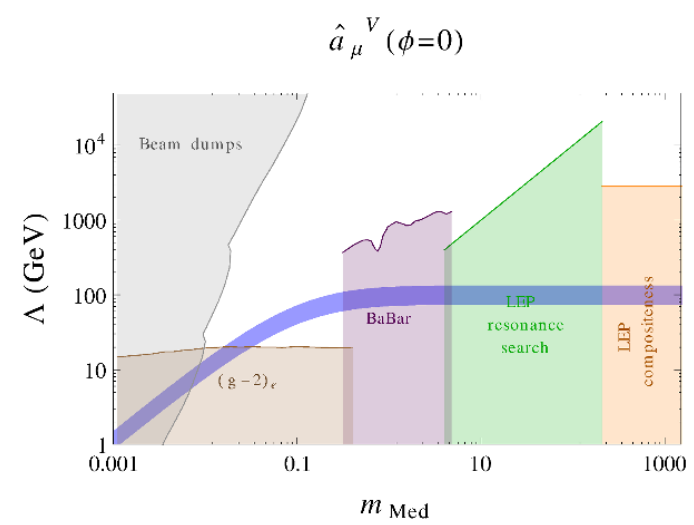

Figure 13. Shown are the favored values which set $\Delta a_{\mu}=0$ to within $2 \sigma$ (blue band) for the neutral vector mediator for Majorana DM.

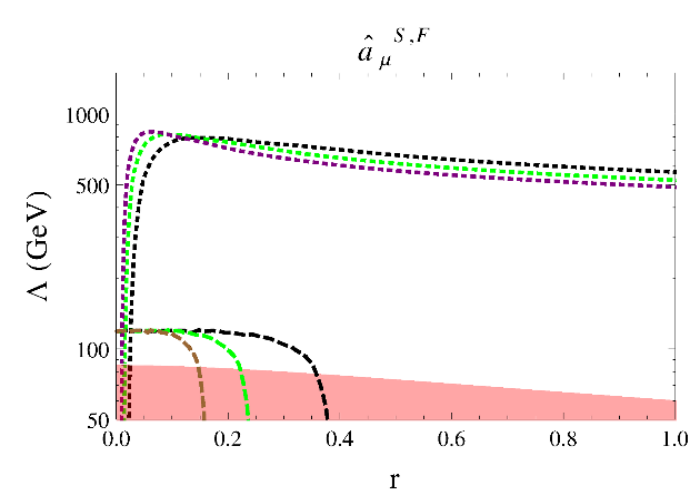

(a)

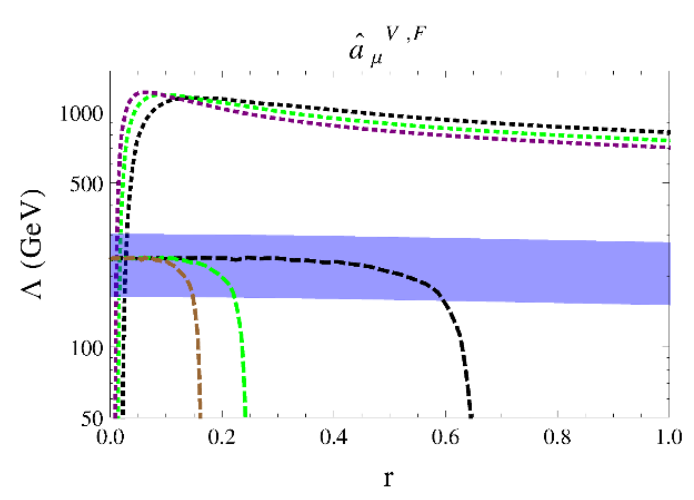

(b)

Figure 14. Plots as a function of $r=m_{\mathrm{DM}} / m_{\mathrm{Med}}$ the $g-2$ analysis of Dirac DM with a charged (a) scalar and (b) vector mediator. In (a) we plot the bound requiring the contribution to $a_{\mu}$ be within $2 \sigma$ of 0 . In (b) we show the region of parameter space favored to set $\Delta a_{\mu}=0$ to within $2 \sigma$. These regions are compared with the bounds from direct detection (dotted) and LEP (dashed). These bounds are shown for 250 (black), 400 (green), and $600 \mathrm{GeV}$ (brown) mediators.

\subsubsection{Neutral spin-1 mediator}

If the interaction is mediated by a neutral massive spin-1 particle $V^{\nu}$, we have for the interaction Lagrangian

$$
\mathcal{L}=\lambda_{V} \bar{\chi} \gamma_{\nu} \gamma^{5} \chi V^{\nu}+\frac{\lambda}{2} \bar{\mu} \gamma^{\mu}\left(\cos \phi+\sin \phi \gamma^{5}\right) \mu V_{\mu}
$$

The results are identical to those for a neutral spin-1 mediator coupling to complex scalar DM, except that the direct detection constraints from that case no longer apply. We plot the results in figure 13. For axial-vector couplings, the results derived for complex scalar DM apply directly, and are shown in figure 10(a). 


\subsection{Dirac DM}

\subsubsection{Charged spin-0 mediator}

In the case of a charged scalar mediator the interaction Lagrangian takes the form

$$
\mathcal{L}=\frac{\lambda}{2}\left[\bar{\chi}\left(1+\gamma_{5}\right) \ell \phi_{\ell}+\bar{\ell}\left(1-\gamma_{5}\right) \chi \phi_{\ell}^{\dagger}\right]
$$

with $\ell$ a lepton and $\phi_{\ell}$ a heavy charged scalar.

The contribution to $g-2$ can be obtained from (2.6), and leads to the relation

$$
\left(\frac{|\lambda|}{m_{\phi}^{2}}\right)^{2}=-\frac{96 \pi^{2} \hat{a}_{\mu}^{S, F}(1-r)^{4}}{m_{\mu}^{2}\left(1-6 r^{2}+3 r^{4}+2 r^{6}-6 r^{4} \ln r^{2}\right)}
$$

where $r=m_{\chi} / m_{\phi}$. Although the sign of the contribution implies that this theory cannot explain the discrepancy in $a_{\mu}$, it can be used to set bounds on the allowed parameter space. These limits are compared with those derived from LEP monophoton searches (dashed curves) and direct detection (dotted curve) in figure 14a. The color of the curves denotes the mass of the mediator, the labelling being $250 \mathrm{GeV}$ (black), $400 \mathrm{GeV}$ (green), or $600 \mathrm{GeV}$ (brown). Additionally, the right-handed slepton bounds from LEP and the LHC also apply, and are shown in figure 11b.

The constants $c$ and $d$ in (3.11) and (3.12) were determined in [37] by matching from the full theory. They lead to

$$
\widetilde{\lambda}^{S, F}=\frac{\lambda^{2} \alpha}{16 \pi m_{\phi}^{2}}\left(1+\frac{2}{3} \sum_{\ell} \ln \frac{m_{\ell}^{2}}{m_{\phi}^{2}} .\right)
$$

A calculation in the effective theory with only the mediator integrated out (performed for this model in [27]) suffices to determine the leading $\log$ behavior of $\widetilde{\lambda}$, and is a very good approximation to the full result.

We see from the figure that the direct detection bounds are much stronger than those arising from $a_{\mu}$, except in the region of very small DM masses, where the limits from monophoton searches are the strongest.

\subsubsection{Charged spin-1 mediator}

If the mediator is a charged vector the interaction given by

$$
\mathcal{L}=\frac{\lambda}{2}\left[\bar{\chi} \gamma_{\mu}\left(1+\gamma_{5}\right) \ell V_{\ell}^{\mu}+\bar{\ell} \gamma_{\mu}\left(1+\gamma_{5}\right) \chi V_{\ell}^{\mu \dagger}\right]
$$

The contribution to the anomalous magnetic moment of the muon can be obtained from (2.4), and leads to the relation

$$
\left(\frac{|\lambda|}{m_{V}^{2}}\right)^{2}=\frac{96 \pi^{2} \hat{a}_{\mu}^{V, F}(1-r)^{4}}{m_{\mu}^{2}\left(10-43 r^{2}+78 r^{4}-49 r^{6}+4 r^{8}+18 r^{6} \ln r^{2}\right)}
$$

where $r=m_{\chi} / m_{V}$. This can be used to determine the region of parameter space favored to explain the anomaly in $a_{\mu}$. 


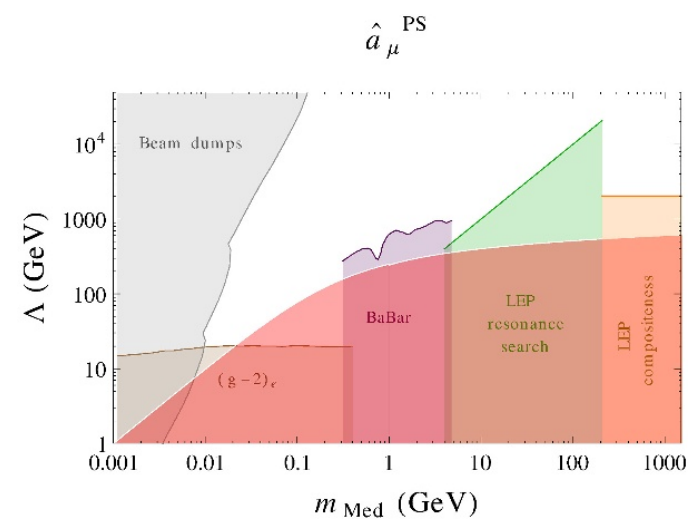

Figure 15. We show disfavored regions of parameter space for Dirac fermion DM with a neutral pseudoscalar mediator as a function of $m_{\text {Med }}$. The shaded exclusion region is drawn by requiring the contributions to $a_{\mu}$ to be less than $2 \sigma$.

For direct detection, the scattering amplitude in the leading logarithm approximation is found to be

$$
\mathcal{M}=\frac{\lambda^{2} \alpha}{12 \pi m_{V}^{2}}\left[\ln \frac{m_{\tau}^{2}}{m_{V}^{2}}+\ln \frac{m_{\mu}^{2}}{m_{V}^{2}}+\ln \frac{|k|^{2}}{m_{V}^{2}}\right] \sum_{q}\left\langle N_{f}\left|Q \bar{q} \gamma^{\nu} q\right| N_{i}\right\rangle \bar{u}\left(p_{2}\right) \gamma_{\nu}\left(1+\gamma^{5}\right) u\left(p_{1}\right)
$$

where as before the $\gamma^{\nu} \gamma_{5}$ terms is velocity suppressed so we neglect it. We therefore obtain

$$
\tilde{\lambda}^{V, F}=\frac{\lambda^{2} \alpha}{12 \pi m_{V}^{2}}\left[\ln \frac{m_{\tau}^{2}}{m_{V}^{2}}+\ln \frac{m_{\mu}^{2}}{m_{V}^{2}}+\ln \frac{|k|^{2}}{m_{V}^{2}}\right] .
$$

The bounds from direct detection (dotted) and LEP monophoton searches (dashed) are compared to this favored region in figure 14b. The limits are plotted for 250 (black), 400 (green), and $600 \mathrm{GeV}$ (brown) mediators. We see that values of $\Lambda$ between $165 \mathrm{GeV}$ and $285 \mathrm{GeV}$ can explain the anomaly in $a_{\mu}$. However, the LEP and direct detection bounds for this range of mediator masses leaves only $r$ values smaller than 0.05 unrestricted, so that the DM mass is favored to be less than about $10 \mathrm{GeV}$.

\subsubsection{Neutral spin-0 mediator}

When the scattering of DM off leptons is mediated by a neutral spin-0 particle $S$ the interaction Lagrangian takes the form

$$
\mathcal{L}_{S}=\lambda \bar{\mu} \mu S \quad \mathcal{L}_{P S}=i \lambda \bar{\mu} \gamma^{5} \mu S
$$

Since the neutral spin-0 mediator does not mix with the photon, the results obtained for the case of real scalar DM summarized in figure 8 continue to apply. The Dirac fermion DM can also couple to leptons via a pseudoscalar. The corresponding bounds for this interaction are shown in figure 15. 


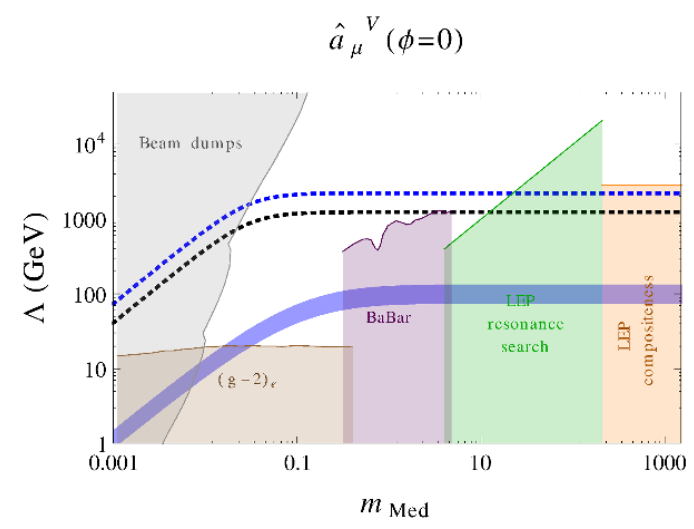

Figure 16. We show the region of parameter space favored by setting $\Delta a_{\mu}=0$ to within $2 \sigma$ (blue shaded) for Dirac DM mediated by a neutral vector. The dotted lines show the direct detection bounds for a DM mass of $10 \mathrm{GeV}$ (blue) and $90 \mathrm{GeV}$ (black).

\subsubsection{Neutral spin-1 mediator}

When the interaction is mediated by a neutral spin- 1 particle the relevant part of the Lagrangian takes the form

$$
\mathcal{L}=\frac{\lambda_{\chi}}{2} \bar{\chi} \gamma^{\nu}\left(\cos \theta+\sin \theta \gamma^{5}\right) \chi V_{\nu}+\frac{\lambda}{2} \bar{\mu} \gamma^{\nu}\left(\cos \phi+\sin \phi \gamma^{5}\right) \mu V_{\nu}
$$

where the mixing angle $\theta$ parametrizes the relative strengths of the vector and axial-vector components of the DM interaction. The $a_{\mu}$ bounds only depend on $\phi$ and not on $\theta$. The direct detection and monophoton limits, on the other hand, depend on both $\theta$ and $\phi$. As before, we pick the pure vector $(\theta=\phi=0)$ and the pure axial vector $(\theta=\phi=\pi / 2)$ cases for illustration. The axial-vector mediator does not mix with the photon, and so the direct detection bounds do not apply. Consequently, the limits are the same as for the complex scalar DM model shown in figure 10(a).

For the vector-vector interaction, the leading logarithmically enhanced contribution to the direct detection scattering amplitude takes the form

$$
\widetilde{\lambda}^{V}=\frac{\lambda^{2} \alpha}{3 \pi m_{V}^{2}}\left[\ln \frac{m_{\tau}^{2}}{\Lambda_{V}^{2}}+\ln \frac{m_{\mu}^{2}}{\Lambda_{V}^{2}}+\ln \frac{|k|^{2}}{\Lambda_{V}^{2}}\right] .
$$

where $\Lambda_{V}$ is the scale of new physics below which the mixing between the mediator and photon is generated. For concreteness we take $\Lambda_{V}=1 \mathrm{TeV}$. The region favored by the muon $g-2$ measurement, and constraints from direct detection and colliders is shown in figure 16 . For heavy mediator masses $\left(m_{M e d}>208 \mathrm{GeV}\right)$, LEP compositeness bounds rule out the entire region consistent with the muon $g-2$ measurement. For light mediators, direct detection constraints are the most severe. These bounds can be avoided for DM masses below direct detection experiment sensitivity $\left(m_{D M}<5 \mathrm{GeV}\right)$, which leaves a window around for mediator masses between about $10 \mathrm{MeV}$ and $300 \mathrm{MeV}$. 


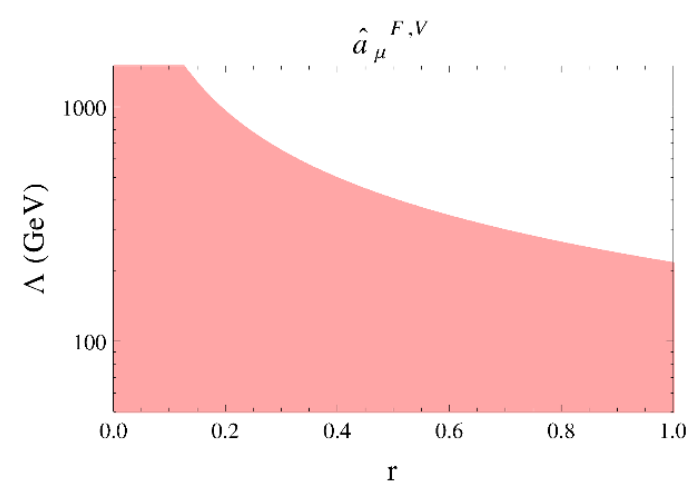

Figure 17. We plot the bound set by requiring the contribution to $a_{\mu}$ by real vector DM mediated by a charged fermion be smaller than $2 \sigma$, as a function of $r=m_{\mathrm{DM}} / m_{\mathrm{Med}}$.

\subsection{Real vector DM}

\subsubsection{Charged fermion mediator}

We conclude our analysis by considering real vector DM candidate $\chi^{\nu}$. If the interactions of DM with leptons are mediated by a charged fermion we obtain the Lagrangian

$$
\mathcal{L}=\frac{\lambda}{2} \bar{\mu} \gamma_{\nu}\left(1+\gamma^{5}\right) F \chi^{\nu}+\text { h.c. }
$$

The contribution to $a_{\mu}$ for this case can be determined from (2.5). This leads to

$$
\left(\frac{|\lambda|}{m_{F}}\right)^{2}=-\frac{96 \pi^{2} r^{2}(1-r)^{4} \hat{a}_{\mu}^{F, V}}{m_{\mu}^{2}\left(5-14 r^{2}+39 r^{4}-38 r^{6}+8 r^{8}+8 r^{4} \ln r^{2}\right)}
$$

with $r=m_{\chi} / m_{F}$. We see from the sign of the contribution that this theory cannot explain the observed discrepancy in $a_{\mu}$.

This scenario is not expected to be significantly constrained by the current limits from direct detection. The monophoton analyses in the literature are also not applicable to this model. We plot the bound obtained by from $a_{\mu}$ in figure 17 as a function of $r$. We find that for small values of $r$ the value of $\Lambda$ is constrained to be fairly large. The weakest bound, around $220 \mathrm{GeV}$, lies close to $r=1$.

\subsubsection{Neutral spin-0 mediator}

When mediation occurs through a neutral spin-0 scalar $S$ we have the Lagrangian

$$
\mathcal{L}_{S}=\lambda \bar{\mu} \mu S
$$

There have been no monophoton studies performed on this model. There are also no significant constraints on this model from direct detection. Therefore, the analysis of a spin-0 mediator coupling to real scalar DM summarized in figure 8 also applies to this case. There are no $C P$-conserving renormalizable couplings of a pseudoscalar mediator with real vector DM. 


\section{Conclusions}

Within the WIMP paradigm, leptophilic DM offers a simple way to remain consistent with current collider, direct detection and indirect detection bounds. In such a scenario, the anomalous magnetic moment of the muon receives corrections from its interactions with dark matter. This can potentially explain the observed discrepancy between the experimentally measured value and the SM prediction. From our analysis, it follows that for many of the simplest leptophilic DM models, a large part of the parameter space which explains the anomaly is excluded by other experiments. Nevertheless, there do exist limited regions of parameter space in these simple theories that can explain the observed anomaly while remaining consistent with current experimental bounds.

For some theories of leptophilic DM, the contribution to $a_{\mu}$ has the wrong sign to explain the anomaly. This can be used to place limits on the parameter space of these theories. We find that, at present, these limits are competitive with those from collider and direct detection experiments.

Note added. While we were completing this paper, we received the manuscripts $[113$, 114] which overlap with some of the ideas presented here.

\section{Acknowledgments}

We would like to thank Brian Batell, Patrick Fox, Brian Hamilton and Samuel McDermott for useful discussions. PA would like to acknowledge support by the National Science Foundation under Grant No. PHYS-1066293 and the hospitality of the Aspen Center for Physics, where a part of this work was completed. ZC and CV are supported by the NSF grant PHY-0968854. Fermilab is operated by Fermi Research Alliance, LLC under Contract No. DE-AC02-07CH11359 with the United States Department of Energy.

Open Access. This article is distributed under the terms of the Creative Commons Attribution License (CC-BY 4.0), which permits any use, distribution and reproduction in any medium, provided the original author(s) and source are credited.

\section{References}

[1] Particle Data Group collaboration, J. Beringer et al., Review of particle physics (RPP), Phys. Rev. D 86 (2012) 010001 [inSPIRE].

[2] CMS collaboration, Observation of a new boson at a mass of $125 \mathrm{GeV}$ with the CMS experiment at the LHC, Phys. Lett. B 716 (2012) 30 [arXiv:1207.7235] [INSPIRE].

[3] ATLAS collaboration, Observation of a new particle in the search for the Standard Model Higgs boson with the ATLAS detector at the LHC, Phys. Lett. B 716 (2012) 1 [arXiv:1207.7214] [INSPIRE].

[4] Planck collaboration, P.A.R. Ade et al., Planck 2013 results. XVI. Cosmological parameters, Astron. Astrophys. (2014) [arXiv:1303.5076] [INSPIRE].

[5] B.W. Lee and S. Weinberg, Cosmological lower bound on heavy neutrino masses, Phys. Rev. Lett. 39 (1977) 165 [INSPIRE]. 
[6] M.I. Vysotsky, A.D. Dolgov and Y. Zeldovich, Cosmological restriction on neutral lepton masses, JETP Lett. 26 (1977) 188 [Pisma Zh. Eksp. Teor. Fiz. 26 (1977) 200] [InSPIRE].

[7] S. Nussinov, Technocosmology: could a technibaryon excess provide a 'natural' missing mass candidate?, Phys. Lett. B 165 (1985) 55 [INSPIRE].

[8] G.B. Gelmini, L.J. Hall and M.J. Lin, What is the cosmion?, Nucl. Phys. B 281 (1987) 726 [INSPIRE].

[9] D.N. Spergel and W.H. Press, Effect of hypothetical, weakly interacting, massive particles on energy transport in the solar interior, Astrophys. J. 294 (1985) 663 [INSPIRE].

[10] S.M. Barr, R.S. Chivukula and E. Farhi, Electroweak fermion number violation and the production of stable particles in the early universe, Phys. Lett. B 241 (1990) 387 [INSPIRE].

[11] S.M. Barr, Baryogenesis, sphalerons and the cogeneration of dark matter, Phys. Rev. D 44 (1991) 3062 [INSPIRE].

[12] D.B. Kaplan, A single explanation for both the baryon and dark matter densities, Phys. Rev. Lett. 68 (1992) 741 [INSPIRE].

[13] S.B. Gudnason, C. Kouvaris and F. Sannino, Towards working technicolor: effective theories and dark matter, Phys. Rev. D 73 (2006) 115003 [hep-ph/0603014] [INSPIRE].

[14] S.B. Gudnason, C. Kouvaris and F. Sannino, Dark matter from new technicolor theories, Phys. Rev. D 74 (2006) 095008 [hep-ph/0608055] [INSPIRE].

[15] R. Kitano and I. Low, Dark matter from baryon asymmetry, Phys. Rev. D 71 (2005) 023510 [hep-ph/0411133] [INSPIRE].

[16] R. Kitano and I. Low, Grand unification, dark matter, baryon asymmetry and the small scale structure of the universe, hep-ph/0503112 [INSPIRE].

[17] D.E. Kaplan, M.A. Luty and K.M. Zurek, Asymmetric dark matter, Phys. Rev. D 79 (2009) 115016 [arXiv:0901.4117] [INSPIRE].

[18] K.M. Zurek, Asymmetric dark matter: theories, signatures and constraints, Phys. Rept. 537 (2014) 91 [arXiv: 1308.0338] [INSPIRE].

[19] L.M. Krauss, S. Nasri and M. Trodden, A model for neutrino masses and dark matter, Phys. Rev. D 67 (2003) 085002 [hep-ph/0210389] [INSPIRE].

[20] E.A. Baltz and L. Bergstrom, Detection of leptonic dark matter, Phys. Rev. D 67 (2003) 043516 [hep-ph/0211325] [INSPIRE].

[21] T. Hambye, K. Kannike, E. Ma and M. Raidal, Emanations of dark matter: muon anomalous magnetic moment, radiative neutrino mass and novel leptogenesis at the $\mathrm{TeV}$ scale, Phys. Rev. D 75 (2007) 095003 [hep-ph/0609228] [INSPIRE].

[22] C.-R. Chen and F. Takahashi, Cosmic rays from leptonic dark matter, JCAP 02 (2009) 004 [arXiv:0810.4110] [InSPIRE].

[23] P.J. Fox and E. Poppitz, Leptophilic dark matter, Phys. Rev. D 79 (2009) 083528 [arXiv:0811.0399] [INSPIRE].

[24] M. Cirelli, M. Kadastik, M. Raidal and A. Strumia, Model-independent implications of the $e^{ \pm}$, anti-proton cosmic ray spectra on properties of dark matter, Nucl. Phys. B 813 (2009) 1 [Addendum ibid. B $\mathbf{8 7 3}$ (2013) 530] [arXiv:0809.2409] [INSPIRE].

[25] A. Ibarra, A. Ringwald, D. Tran and C. Weniger, Cosmic rays from leptophilic dark matter decay via kinetic mixing, JCAP 08 (2009) 017 [arXiv:0903.3625] [INSPIRE]. 
[26] H. Davoudiasl, Dark matter with time-varying leptophilic couplings, Phys. Rev. D 80 (2009) 043502 [arXiv: 0904.3103] [INSPIRE].

[27] J. Kopp, V. Niro, T. Schwetz and J. Zupan, DAMA/LIBRA and leptonically interacting dark matter, Phys. Rev. D 80 (2009) 083502 [arXiv:0907.3159] [INSPIRE].

[28] T. Cohen and K.M. Zurek, Leptophilic dark matter from the lepton asymmetry, Phys. Rev. Lett. 104 (2010) 101301 [arXiv:0909.2035] [INSPIRE].

[29] D. Spolyar, M.R. Buckley, K. Freese, D. Hooper and H. Murayama, High energy neutrinos as a test of leptophilic dark matter, arXiv:0905.4764 [INSPIRE].

[30] H.-S. Goh, L.J. Hall and P. Kumar, The leptonic Higgs as a messenger of dark matter, JHEP 05 (2009) 097 [arXiv: 0902.0814] [INSPIRE].

[31] Q.-H. Cao, E. Ma and G. Shaughnessy, Dark matter: the leptonic connection, Phys. Lett. B 673 (2009) 152 [arXiv:0901.1334] [INSPIRE].

[32] P. Ko and Y. Omura, Supersymmetric $\mathrm{U}(1)_{B} \times \mathrm{U}(1)_{L}$ model with leptophilic and leptophobic cold dark matters, Phys. Lett. B 701 (2011) 363 [arXiv:1012.4679] [InSPIRE].

[33] W. Chao, Pure leptonic gauge symmetry, neutrino masses and dark matter, Phys. Lett. B 695 (2011) 157 [arXiv: 1005.1024] [INSPIRE].

[34] W.-Z. Feng, P. Nath and G. Peim, Cosmic coincidence and asymmetric dark matter in a Stückelberg extension, Phys. Rev. D 85 (2012) 115016 [arXiv:1204.5752] [INSPIRE].

[35] D. Schmidt, T. Schwetz and T. Toma, Direct detection of leptophilic dark matter in a model with radiative neutrino masses, Phys. Rev. D 85 (2012) 073009 [arXiv:1201.0906] [INSPIRE].

[36] M. Das and S. Mohanty, Leptophilic dark matter in gauged $L_{\mu}-L_{\tau}$ extension of MSSM, Phys. Rev. D 89 (2014) 025004 [arXiv: 1306.4505] [INSPIRE].

[37] P. Agrawal, S. Blanchet, Z. Chacko and C. Kilic, Flavored dark matter and its implications for direct detection and colliders, Phys. Rev. D 86 (2012) 055002 [arXiv:1109.3516] [INSPIRE].

[38] A. Crivellin, F. D'Eramo and M. Procura, New constraints on dark matter effective theories from standard model loops, Phys. Rev. Lett. 112 (2014) 191304 [arXiv:1402.1173] [INSPIRE].

[39] M. Papucci and A. Strumia, Robust implications on dark matter from the first FERMI sky gamma map, JCAP 03 (2010) 014 [arXiv:0912.0742] [INSPIRE].

[40] K.N. Abazajian, P. Agrawal, Z. Chacko and C. Kilic, Conservative constraints on dark matter from the Fermi-LAT isotropic diffuse gamma-ray background spectrum, JCAP 11 (2010) 041 [arXiv: 1002.3820] [INSPIRE].

[41] G. Hutsi, A. Hektor and M. Raidal, Implications of the Fermi-LAT diffuse gamma-ray measurements on annihilating or decaying dark matter, JCAP 07 (2010) 008 [arXiv: 1004.2036] [INSPIRE].

[42] K.N. Abazajian, S. Blanchet and J.P. Harding, Current and future constraints on dark matter from prompt and inverse-Compton photon emission in the isotropic diffuse gamma-ray background, Phys. Rev. D 85 (2012) 043509 [arXiv: 1011. 5090] [INSPIRE].

[43] D. Hooper and T. Linden, On the origin of the gamma rays from the galactic center, Phys. Rev. D 84 (2011) 123005 [arXiv:1110.0006] [INSPIRE]. 
[44] S. Blanchet and J. Lavalle, Diffuse gamma-ray constraints on dark matter revisited. I: the impact of subhalos, JCAP 11 (2012) 021 [arXiv: 1207.2476] [INSPIRE].

[45] D. Hooper, C. Kelso and F.S. Queiroz, Stringent and robust constraints on the dark matter annihilation cross section from the region of the galactic center, Astropart. Phys. 46 (2013) 55 [arXiv: 1209.3015] [INSPIRE].

[46] M. Tavakoli, I. Cholis, C. Evoli and P. Ullio, Constraints on dark matter annihilations from diffuse gamma-ray emission in the galaxy, JCAP 01 (2014) 017 [arXiv:1308.4135] [INSPIRE].

[47] I. Cholis, D. Hooper and S.D. McDermott, Dissecting the gamma-ray background in search of dark matter, JCAP 02 (2014) 014 [arXiv: 1312.0608] [INSPIRE].

[48] Fermi-LAT collaboration, M. Ackermann et al., Dark matter constraints from observations of 25 milky way satellite galaxies with the Fermi Large Area Telescope, Phys. Rev. D 89 (2014) 042001 [arXiv:1310.0828] [INSPIRE].

[49] J. Goodman et al., Gamma ray line constraints on effective theories of dark matter, Nucl. Phys. B 844 (2011) 55 [arXiv: 1009.0008] [inSPIRE].

[50] K.N. Abazajian, P. Agrawal, Z. Chacko and C. Kilic, Lower limits on the strengths of gamma ray lines from WIMP dark matter annihilation, Phys. Rev. D 85 (2012) 123543 [arXiv:1111.2835] [INSPIRE].

[51] T. Bringmann, X. Huang, A. Ibarra, S. Vogl and C. Weniger, Fermi-LAT search for internal bremsstrahlung signatures from dark matter annihilation, JCAP 07 (2012) 054 [arXiv: 1203.1312] [INSPIRE].

[52] T. Bringmann and C. Weniger, Gamma ray signals from dark matter: concepts, status and prospects, Phys. Dark Univ. 1 (2012) 194 [arXiv:1208.5481] [INSPIRE].

[53] B. Shakya, A 130 GeV gamma ray signal from supersymmetry, Phys. Dark Univ. 2 (2013) 83 [arXiv:1209.2427] [INSPIRE].

[54] L. Bergstrom, The $130 \mathrm{GeV}$ fingerprint of right-handed neutrino dark matter, Phys. Rev. D 86 (2012) 103514 [arXiv:1208.6082] [INSPIRE].

[55] M. Garny, A. Ibarra, M. Pato and S. Vogl, Internal bremsstrahlung signatures in light of direct dark matter searches, JCAP 12 (2013) 046 [arXiv:1306.6342] [INSPIRE].

[56] T. Toma, Internal bremsstrahlung signature of real scalar dark matter and consistency with thermal relic density, Phys. Rev. Lett. 111 (2013) 091301 [arXiv:1307.6181] [INSPIRE].

[57] F. Giacchino, L. Lopez-Honorez and M.H.G. Tytgat, Scalar dark matter models with significant internal bremsstrahlung, JCAP 10 (2013) 025 [arXiv:1307.6480] [INSPIRE].

[58] J. Kopp, L. Michaels and J. Smirnov, Loopy constraints on leptophilic dark matter and internal bremsstrahlung, JCAP 04 (2014) 022 [arXiv:1401.6457] [INSPIRE].

[59] B. Kyae, PAMELA/ATIC anomaly from the meta-stable extra dark matter component and the leptophilic Yukawa interaction, JCAP 07 (2009) 028 [arXiv:0902.0071] [INSPIRE].

[60] X.-J. Bi, X.-G. He and Q. Yuan, Parameters in a class of leptophilic models from PAMELA, ATIC and FERMI, Phys. Lett. B 678 (2009) 168 [arXiv:0903.0122] [INSPIRE].

[61] N. Haba, Y. Kajiyama, S. Matsumoto, H. Okada and K. Yoshioka, Universally leptophilic dark matter from non-Abelian discrete symmetry, Phys. Lett. B 695 (2011) 476 [arXiv: 1008.4777] [INSPIRE]. 
[62] C.D. Carone and R. Primulando, A Froggatt-Nielsen model for leptophilic scalar dark matter decay, Phys. Rev. D 84 (2011) 035002 [arXiv:1105.4635] [InSPIRE].

[63] L. Bergstrom, T. Bringmann, I. Cholis, D. Hooper and C. Weniger, New limits on dark matter annihilation from AMS cosmic ray positron data, Phys. Rev. Lett. 111 (2013) 171101 [arXiv: 1306.3983] [INSPIRE].

[64] A. Ibarra, A.S. Lamperstorfer and J. Silk, Dark matter annihilations and decays after the AMS-02 positron measurements, Phys. Rev. D 89 (2014) 063539 [arXiv:1309.2570] [INSPIRE].

[65] K. Fukushima and J. Kumar, Dipole moment bounds on dark matter annihilation, Phys. Rev. D 88 (2013) 056017 [arXiv:1307.7120] [INSPIRE].

[66] T. Kinoshita and M. Nio, The tenth-order QED contribution to the lepton $g-2$ : evaluation of dominant $\alpha^{5}$ terms of muon $g-2$, Phys. Rev. D 73 (2006) 053007 [hep-ph/0512330] [INSPIRE].

[67] A. Czarnecki, W.J. Marciano and A. Vainshtein, Refinements in electroweak contributions to the muon anomalous magnetic moment, Phys. Rev. D 67 (2003) 073006 [Erratum ibid. D 73 (2006) 119901] [hep-ph/0212229] [INSPIRE].

[68] K. Hagiwara, R. Liao, A.D. Martin, D. Nomura and T. Teubner, $(g-2)_{\mu}$ and $\alpha\left(M_{Z}^{2}\right)$ re-evaluated using new precise data, J. Phys. G 38 (2011) 085003 [arXiv:1105.3149] [INSPIRE].

[69] M. Davier and W.J. Marciano, The theoretical prediction for the muon anomalous magnetic moment, Ann. Rev. Nucl. Part. Sci. 54 (2004) 115 [INSPIRE].

[70] M. Passera, The standard model prediction of the muon anomalous magnetic moment, J. Phys. G 31 (2005) R75 [hep-ph/0411168] [InSPIRE].

[71] Muon G-2 collaboration, G.W. Bennett et al., Final report of the muon E821 anomalous magnetic moment measurement at BNL, Phys. Rev. D 73 (2006) 072003 [hep-ex/0602035] [INSPIRE].

[72] M. Davier, S. Eidelman, A. Hocker and Z. Zhang, Updated estimate of the muon magnetic moment using revised results from $e^{+} e^{-}$annihilation, Eur. Phys. J. C 31 (2003) 503 [hep-ph/0308213] [INSPIRE].

[73] I.R. Blokland, A. Czarnecki and K. Melnikov, Pion pole contribution to hadronic light by light scattering and muon anomalous magnetic moment, Phys. Rev. Lett. 88 (2002) 071803 [hep-ph/0112117] [INSPIRE].

[74] K. Melnikov and A. Vainshtein, Hadronic light-by-light scattering contribution to the muon anomalous magnetic moment revisited, Phys. Rev. D 70 (2004) 113006 [hep-ph/0312226] [INSPIRE].

[75] J.F. de Troconiz and F.J. Yndurain, The hadronic contributions to the anomalous magnetic moment of the muon, Phys. Rev. D 71 (2005) 073008 [hep-ph/0402285] [INSPIRE].

[76] J. Bijnens and J. Prades, The hadronic light-by-light contribution to the muon anomalous magnetic moment: where do we stand?, Mod. Phys. Lett. A 22 (2007) 767 [hep-ph/0702170] [INSPIRE].

[77] M. Davier, A. Hoecker, B. Malaescu and Z. Zhang, Reevaluation of the hadronic contributions to the muon $g-2$ and to $\alpha\left(M_{Z}^{2}\right)$, Eur. Phys. J. C 71 (2011) 1515 [Erratum ibid. C 72 (2012) 1874] [arXiv:1010.4180] [INSPIRE]. 
[78] J.P. Miller, E.d. Rafael, B.L. Roberts and D. Stöckinger, Muon $(g-2)$ : experiment and theory, Ann. Rev. Nucl. Part. Sci. 62 (2012) 237 [InSPIRE].

[79] F. Jegerlehner and A. Nyffeler, The muon g-2, Phys. Rept. 477 (2009) 1 [arXiv:0902.3360] [INSPIRE].

[80] D. Hanneke, S. Fogwell and G. Gabrielse, New measurement of the electron magnetic moment and the fine structure constant, Phys. Rev. Lett. 100 (2008) 120801 [arXiv:0801.1134] [INSPIRE].

[81] J.P. Leveille, The second order weak correction to $(g-2)$ of the muon in arbitrary gauge models, Nucl. Phys. B 137 (1978) 63 [INSPIRE].

[82] S.R. Moore, K. Whisnant and B.-L. Young, Second order corrections to the muon anomalous magnetic moment in alternative electroweak models, Phys. Rev. D 31 (1985) 105 [InSPIRE].

[83] J.A. Grifols and A. Méndez, Constraints on supersymmetric particle masses from $(g-2)_{\mu}$, Phys. Rev. D 26 (1982) 1809 [INSPIRE].

[84] LUX collaboration, D.S. Akerib et al., First results from the LUX dark matter experiment at the Sanford Underground Research Facility, Phys. Rev. Lett. 112 (2014) 091303 [arXiv:1310.8214] [INSPIRE].

[85] A. Kumar and S. Tulin, Top-flavored dark matter and the forward-backward asymmetry, Phys. Rev. D 87 (2013) 095006 [arXiv:1303.0332] [INSPIRE].

[86] Y. Zeldovich, Electromagnetic interaction with parity violation, Sov. Phys. JETP 6 (1958) 1184 [Zh. Eksp. Teor. Fiz. 33 (1958) 1531].

[87] J.F. Nieves, Electromagnetic properties of Majorana neutrinos, Phys. Rev. D 26 (1982) 3152 [INSPIRE].

[88] B. Kayser, Majorana neutrinos and their electromagnetic properties, Phys. Rev. D 26 (1982) 1662 [INSPIRE].

[89] E.E. Radescu, Comments on the electromagnetic properties of Majorana fermions, Phys. Rev. D 32 (1985) 1266 [INSPIRE].

[90] F. Boudjema, C. Hamzaoui, V. Rahal and H.C. Ren, Electromagnetic properties of generalized Majorana particles, Phys. Rev. Lett. 62 (1989) 852 [INSPIRE].

[91] F. Boudjema and C. Hamzaoui, Massive and massless Majorana particles of arbitrary spin: covariant gauge couplings and production properties, Phys. Rev. D 43 (1991) 3748 [INSPIRE].

[92] V. Barger, W.-Y. Keung and D. Marfatia, Electromagnetic properties of dark matter: dipole moments and charge form factor, Phys. Lett. B 696 (2011) 74 [arXiv:1007.4345] [INSPIRE].

[93] S. Chang, N. Weiner and I. Yavin, Magnetic inelastic dark matter, Phys. Rev. D 82 (2010) 125011 [arXiv: 1007.4200] [INSPIRE].

[94] P.J. Fox, R. Harnik, J. Kopp and Y. Tsai, LEP shines light on dark matter, Phys. Rev. D 84 (2011) 014028 [arXiv: 1103.0240] [InSPIRE].

[95] E. Eichten, K.D. Lane and M.E. Peskin, New tests for quark and lepton substructure, Phys. Rev. Lett. 50 (1983) 811 [INSPIRE].

[96] H. Kroha, Compositeness limits from $e^{+} e^{-}$annihilation revisited, Phys. Rev. D 46 (1992) 58 [INSPIRE]. 
[97] ALEPH, DELPHI, L3, OPAL and LEP Electroweak collaborations, S. Schael et al., Electroweak measurements in electron-positron collisions at W-boson-pair energies at LEP, Phys. Rept. 532 (2013) 119 [arXiv:1302.3415] [INSPIRE].

[98] D. Bourilkov, Hint for axial vector contact interactions in the data on $e^{+} e^{-} \rightarrow e^{+} e^{-}(\gamma)$ at center-of-mass energies 192-208 GeV, Phys. Rev. D 64 (2001) 071701 [hep-ph/0104165] [INSPIRE].

[99] R. Essig et al., Working group report: new light weakly coupled particles, arXiv:1311.0029 [INSPIRE].

[100] E.M. Riordan et al., A search for short lived axions in an electron beam dump experiment, Phys. Rev. Lett. 59 (1987) 755 [INSPIRE].

[101] J.D. Bjorken et al., Search for neutral metastable penetrating particles produced in the SLAC beam dump, Phys. Rev. D 38 (1988) 3375 [InSPIRE].

[102] A. Bross et al., A search for shortlived particles produced in an electron beam dump, Phys. Rev. Lett. 67 (1991) 2942 [INSPIRE].

[103] J.D. Bjorken, R. Essig, P. Schuster and N. Toro, New fixed-target experiments to search for dark gauge forces, Phys. Rev. D 80 (2009) 075018 [arXiv:0906.0580] [INSPIRE].

[104] N. Borodatchenkova, D. Choudhury and M. Drees, Probing MeV dark matter at low-energy $e^{+} e^{-}$colliders, Phys. Rev. Lett. 96 (2006) 141802 [hep-ph/0510147] [INSPIRE].

[105] P. Fayet, $U$-boson production in $e^{+} e^{-}$annihilations, $\psi$ and $\Upsilon$ decays and light dark matter, Phys. Rev. D 75 (2007) 115017 [hep-ph/0702176] [INSPIRE].

[106] M. Reece and L.-T. Wang, Searching for the light dark gauge boson in GeV-scale experiments, JHEP 07 (2009) 051 [arXiv:0904.1743] [INSPIRE].

[107] ATLAS collaboration, Search for direct-slepton and direct-chargino production in final states with two opposite-sign leptons, missing transverse momentum and no jets in $20 \mathrm{fb}^{-1}$ of pp collisions at $\sqrt{s}=8 \mathrm{TeV}$ with the ATLAS detector, ATLAS-CONF-2013-049, CERN, Geneva Switzerland (2013).

[108] P. Agrawal, Z. Chacko, C. Kilic and R.K. Mishra, A classification of dark matter candidates with primarily spin-dependent interactions with matter, arXiv:1003.1912 [INSPIRE].

[109] J. Fan, M. Reece and L.-T. Wang, Non-relativistic effective theory of dark matter direct detection, JCAP 11 (2010) 042 [arXiv: 1008.1591] [INSPIRE].

[110] S. Chang, R. Edezhath, J. Hutchinson and M. Luty, Effective WIMPs, Phys. Rev. D 89 (2014) 015011 [arXiv:1307.8120] [INSPIRE].

[111] Y. Bai and J. Berger, Fermion portal dark matter, JHEP 11 (2013) 171 [arXiv:1308.0612] [INSPIRE].

[112] A. DiFranzo, K.I. Nagao, A. Rajaraman and T.M.P. Tait, Simplified models for dark matter interacting with quarks, JHEP 11 (2013) 014 [arXiv: 1308.2679] [INSPIRE].

[113] Y. Bai and J. Berger, Lepton portal dark matter, arXiv:1402.6696 [INSPIRE].

[114] A. Freitas, J. Lykken, S. Kell and S. Westhoff, Testing the muon $g-2$ anomaly at the LHC, JHEP 05 (2014) 145 [arXiv: 1402.7065] [INSPIRE]. 